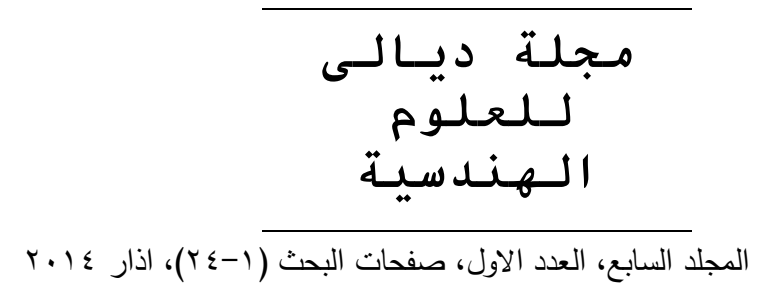

\title{
تقييم الأداء الإنشائي لمشاريع محافظة ديالى باستخدام تقنية تقييم البدائل المتعددة المعايير النسبية المعقدة بعلاقات رمادية (COPRAS-G)
}

\author{
حافظ ابراهيم ناجي \\ مدرس/ كلية الهندسة/ جامعة ديالى/ العراق

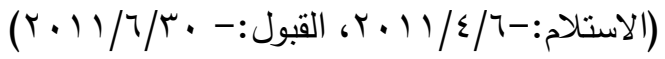

الخلاصة

يعد القطاع الانشائي المحرك الاساسي للاقتصاد الوطني من جهة ومن جهة ثانية يواجه هذا القطاع تعثرا في

اداءه ويمتاز باندماج عدة مشاكل في ان واحد ناتجة عن الطبيعة المعقدة وغير المؤكدة لبيئة المشروع وتعدد العوامل التي تؤدي الى حدوث تاخير زمني في التقفذ وارتفاع في كلفة المشروع، ولمواجهة هذه التحديات يتطلب من ادارة المشروع في هذا المجال طرق وامكانيات ذكية ومميزة في اتخاذ القرارات الصحيحة والصائبة .ويعد اسلوب تقييم البدائل المتعددة المعايير النسبية المعقدة بعلاقات رمادية (COPRAS-G) احد تلك الأساليب. يركز هذا البحث على تقديم كتقنية تساعد على اتخاذ القرار الامتل عند انشاء المشروع الذي تعترضه مشاكل معقدة ومتعدد المعايير (MCDM). وتطبق هذه التقنية على مجموعة متتوعة من المساحات كونها تسمح بالدراسة الشاملة وغير المتحيزة للمعايير والبدائل. يهدف هذا البحث الى ابراز اهمية الاداء الانثائي، وكيف يمكن توظيف اسس تقنية (COPRAS-G) في تقييم الاداء الانشائي لمشاريع محافظة ديالى من خلال تحديد المعايير المستخدمة لتحديد المشروع الأمنل من ناحية الهية الاداء الانشائي، وكذلك تحديد قائمة بمشاريع المحافظة الرئيسة لكي نختار المشروع الامتل من هذه الناحية .ولغرض تحقيق هدف البحث، فقد تم جمع البيانات الخاصة به من الادبيات التي تتاولت موضوعي تقييم البدائل المتعددة المعايير النسبية المعقدة بعلاقات رمادية والاداء الانشائي، واخيرا من المقابلات الشخصية من ذوي الاختصاص من المصممين والمنفذين لهذه المشاريع. اظهرت نتائج تحليل البيانات لافراد العينة ان معايير الكلفة، الوقت، والنوعية هي اكثر المعايير اهية لاجراء المقارنات الثنائية بين المشاريع، وان معيار الكلفة هو اكثر اهية من بقية المعايير في المشاريع .واخيرا كومن خلال حساب الاهمية النسبية للمشاريع واولويات البدائل ودرجة الكفاءة لها نجد ان مشروع محطة مجاري بعقوبة قد حصل على النصيب الاكبر من الأهمية والمنفعة بالمقارنة مع المشاريع الاخرى. وفي النهاية نم التوصل الى مجموعة من الاستتناجات والتوصيات لمختلف جوانب الموضوع من بينها، الإسراع في تطبيق تقنيات اتخاذ القرار المتعدد المعايير في تقييم المشاريع إضافة الى الإسراع في تطبيق النظم المقترحة للأداء

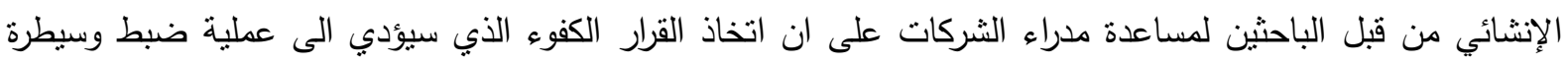
اكثر فعالية على المشروع الانثائي. 
الكلمات الدالة: الاداء الانشائي، طريقة تقييم البدائل المتعددة المعايير النسبية المعقدة بعلاقات رمادية.

$$
\text { - } 1 \text { - المقدمة }
$$

في العديد من القرارات لايمكن التتبؤ بنتائج طرق العمل البديلة بصورة اكيدة ('). مثالها قرار شركة معينة في اطلاق منتج جديد سوف تكون نتائج نجاحه غير مؤكدة، وكذلك قرار المستثر في سوق الاسهم المالية في اختيار

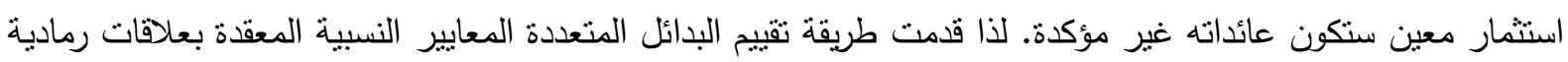
a method of multiple criteria COmplex PRoportional ASsessment of alternatives with Grey relations للتعامل مع مشاكل اتخاذ القرار المتعدد المعايير وبقيم رمادية. ان الفكرة من تطبيق طريقة COPRAS-G ناتي من الظروف الحقيقة في اتخاذ القرارات ومن تطبيقات الانظمة

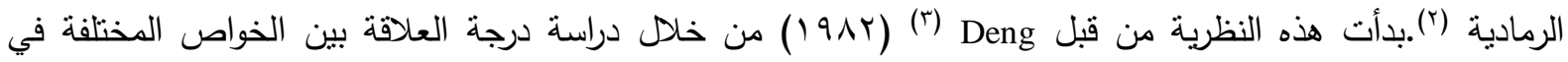

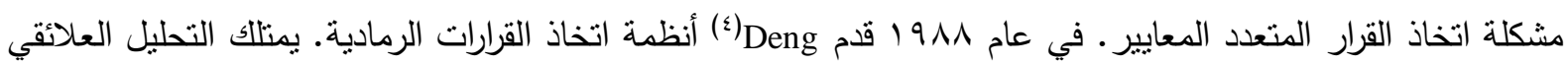
الرمادي فوائد منها (؟): يتضمن حسابات بسيطة، ينطلب عينات اصغر ، عدم الحاجة للتوزيع المثالي من العينات، النتائج

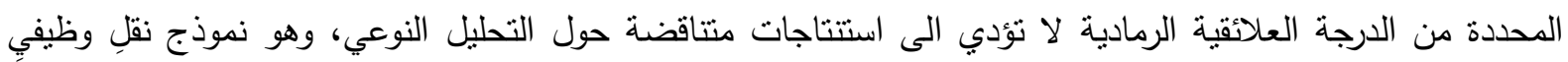

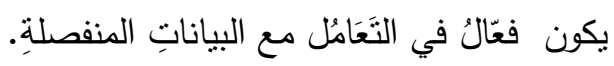
والنظرية مفيدة رياضيا عندما يتم التعامل مع نظام بمعلومات محددة.فطبقا لهذه النظرية، النظام الذي تعرف

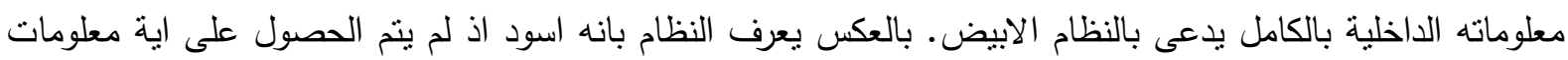

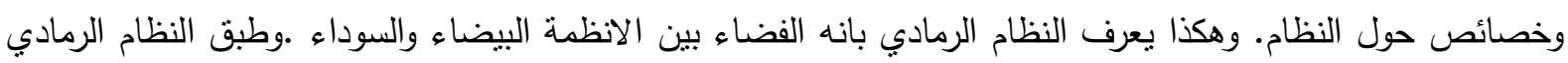

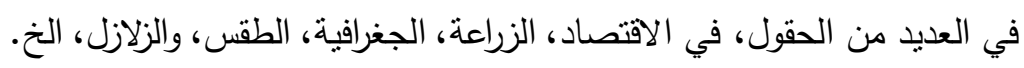

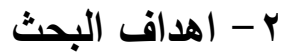

يهدف البحث الى صياغة استراتيجية منظمة تشتمل توظيف تقنية تقييم البدائل المتعددة المعايير النسبية المعقدة بالتكامل

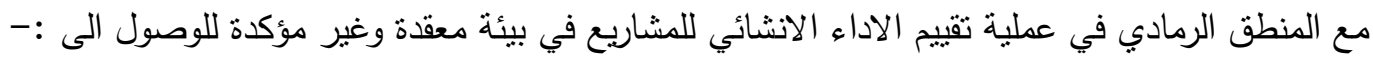
أ- - فهم موضوعي للواقع الإنثائي. ب- - التعرف على المعايير المؤثرة على الأداء الإنثائي. ت- صياغة التقنية الهجينة لاتخاذ القرار الامثل لتقييم الاداء الانثائي وبالتالي معالجة نقاط الضعف فيه. r - خطوات تنفيذ تقتية تقييم البدائل المتعددة المعايير النسبية المعقدة بعلاقات رمادية ان تحديد الأهمية والأولوية ودرجة المنفعة للبدائل تتفذ في هذه الطريقة بالمراحل التالية(؟):-

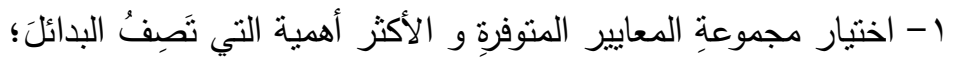
Y- إعداد مصفوفةِ اتخاذ القراراتَ X $X=\left[\begin{array}{llr}{[L 11 ; U 11]} & {[L 12 ; U 12] \cdots} & {[L 1 m ; U 1 m]} \\ {[L 21 ; U 21] \vdots} & {[L 22 ; U 22] \ddots} & \vdots[L 2 m ; U 2 m] \\ {[L n 1 ; U n 1]} & {[L n 2 ; U n 2] \cdots} & {[L n m ; U n m]}\end{array}\right] \quad i=1 ; \ldots . . ; m ; j=1 ; \ldots . . ; n$ 
حيث Lij - القيمة الأقل أو الحدّ الأوطأ للمعيار th في بديل الحل Uij ,i th - القيمة الأكبر أو الحدّ الأعلى للمعيار

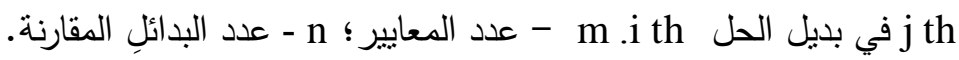

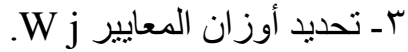

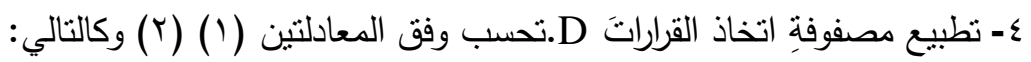

$\overline{L l J}=\frac{L i j}{1 / 2\left(\sum_{j=1}^{n} L i j+\sum_{j=1}^{n} U i j\right)}=\frac{2 L i j}{\left(\sum_{j=1}^{n} L i j+\sum_{j=1}^{n} U i j\right)}$

$\overline{U l J}=\frac{U i j}{1 / 2\left(\sum_{j=1}^{n} L i j+\sum_{j=1}^{n} U i j\right)}=\frac{2 U i j}{\left(\sum_{j=1}^{n} L i j+\sum_{j=1}^{n} U i j\right)}$

$i=1 ; . . . . . ; m ; j=1 ; \ldots . . . . ; n$.

$D=\left[\begin{array}{llr}{[\overline{L 11} ; \overline{U 11}]} & {[\overline{L 12} ; \overline{U 12}] \cdots} & {[\overline{L 1 m} ; \overline{U 1 m}]} \\ {[\overline{L 21} ; \overline{U 21}]:} & {[\overline{L 22} ; \overline{U 22}] \ddots} & \vdots[\overline{L 2 m} ; \overline{U 2 m}] \\ {[\overline{L n 1} ; \overline{U n 1}]} & {[\overline{L n 2} ; \overline{U n 2}] \cdots} & {[\overline{L n m} ; \overline{U n m}]}\end{array}\right]$

وبذلك تكون المصفوفة كما مبين:-

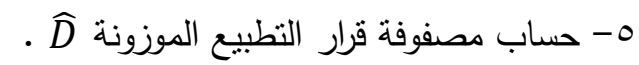

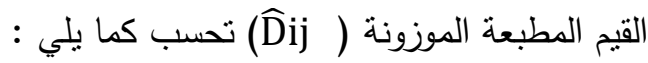

$\widehat{L \imath \jmath}=\overline{L \imath \jmath} \cdot \mathrm{Wj} \quad, \quad \widehat{U \imath \jmath}=\overline{U \imath \jmath} \cdot \mathrm{Wj}$

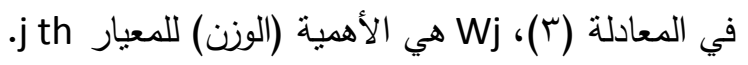
وبذلك تكون مصفوفة اتخاذ القرار المطبعة الموزونة:

$\widehat{D}=\left[\begin{array}{llc}{[\widehat{L 11} ; \widehat{U 11}]} & {[\widehat{L 12} ; \widehat{U 12}] \cdots} & {[\widehat{L 1 m} ; \widehat{U 1 m}]} \\ {[\widehat{L 21} ; \widehat{U 21}]} & {[\widehat{L 22} ; \widehat{U 22}]} & \vdots[\widehat{L 2 m} ; \widehat{U 2 m}] \\ {[\widehat{L n 1} ; \widehat{U n 1}]} & {[\widehat{L n 2} ; \widehat{U n 2}] \cdots} & {[\widehat{L n m} ; \widehat{U n m}]}\end{array}\right]$

إنّ غرضَ هذه المرحلة أَنْ نَنتمَ القيم الموزونة بدون ابعاد من مؤشرات المقارنة . عندما تعرف قيم المؤشرات بدون ابعاد

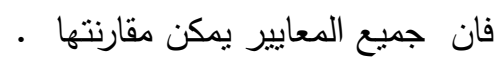

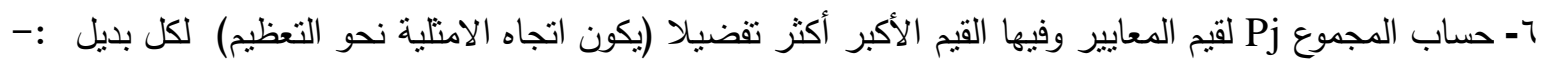
$P j=1 / 2 \sum_{i=1}^{k}(\widehat{L l J}+\widehat{U l} J)$

في المعادلة (ع)، k هي عدد المعايير التي يجب أن تعظم (حيث يفترض اولا وضع المعايير مع تعظيم اتجاه الامثلية في

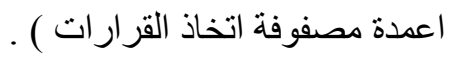
V- حساب المجموع Rj لقيم المعايير وفيها القيم الاصغر اكثر تفضيلا (يكون اتجاه الامنلية التننية) لكل بديل في $R j=1 / 2 \sum_{i=k+1}^{m}(\widehat{L i j}+\widehat{U l})$ مصفوفة اتخاذ القرار : :في الصيغة (0) ، (m - k) هي عدد المعايير التي يجب ان تقلل (يفترض وضع المعايير مع تدنية اتجاه الامثلية في

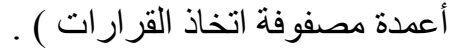
Rj تحديد القيمة الأدنى ل

$\operatorname{Rmin}=\min _{j}^{B j}, j=\overline{J, m}$

$W j=P j+\frac{R m i n \sum_{j=1}^{n} R j}{R j \sum_{j=1}^{n} \frac{R m i n}{R j}} \quad$ gl $\quad W j=P j+\frac{\sum_{j=1}^{n} R j}{R j \sum_{j=1}^{n} \frac{1}{R j}}$

9- حساب الوزن النسبي لكل بديل Wj

K ت الحديد معيار الامثلية

$K=\max _{j} \quad j=\overline{1, n}$ 
ا ـ تحديد أولويةِ المشروعَ. المشروع الذي يمنلك اهمية (وزن نسبي) Wj اعظم ، يكون الاعلى اولوية او رتبة . r ا. حساب درجة المنفعة لكل بديل . حيث تحدد درجة منفعة المشروع بمقارنة المشاريع المحللة مع المشروع الأفضل.

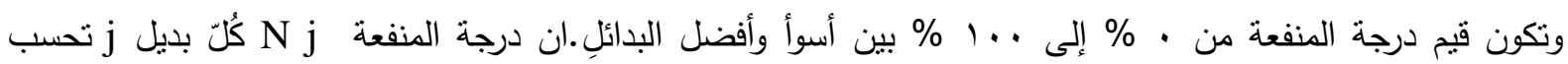
كالأتي :$N_{j=} \frac{W_{j}}{W_{\max }} * 100 \%$

حيث Wj الأهمية النسبية للمشروع j يُشيرُ إلى درجةِ الرضاً لمنطلبات المشاركين في المشروعَ. Wmax ان اسلوب القرار المقترح في هذا المقطع يسمحَ بتقييم الإعتمادِ المبانشِرِ والنسبي لدرجةِ المنفعة وأهميةً البدائلِ في نظام

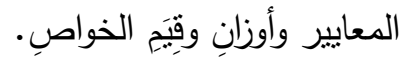

\section{ع - حساب الاهمية للمعاييز}

هنالك العديد من الطرق المستخدمة في حساب الاهمية للمعايير، وفي بحثنا هذا استخدمنا طرق ترتيب النظام، هذه الطرق بسيطة في اعطاء الوزن لعدد من الفقرات مرنبة وفقا لاهميتها. ويمكن ان يرتب متخذوا القرار الخواصَ أَو لهاب المعاييرَ بسهولة اكثر بكثير من ان يعطوا وزنا لها .تاخذ هذه الطرق ذلك الترتيب كمدخلات وتحولها الى اوزان لكل من لهن تلك المعاييزَ اقترح Stillwell (0) في عام (9191اعدة صيغ لاستخراج الوزن النسبي بالاعتماد على نرتيب المعايير في النظام، تدعى الصيخة الاولى مجموع الرتب Rank - Sum)، إنّ الخطوةَ الأولى هيْ ترتيب الخواصَ أَو المعاييرَ

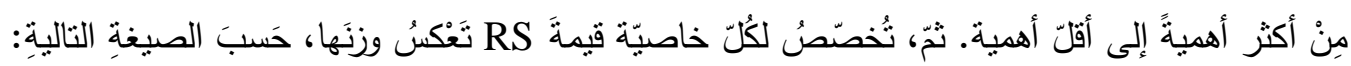
$W i(R S)=\frac{n+1-i}{\sum_{j=1}^{n} j} \quad j=1, \ldots \ldots, n--\cdot-\cdot-\cdot-\cdot-\cdot-\cdot-\cdot-\cdot-\cdot-\cdot-\cdot--(1$.

Reciprocal of the وباسلوب مشابه نستخرج الوزن النسبي بالاعتماد على الصيغة الثانية والتي تدعى بالترتيب المتبادل: RR :(RR) Ranks $W i(R S)=\frac{\frac{1}{i}}{\sum_{i=1}^{n} \frac{1}{j}}$ $j=1$,

واقتزح كل من Barron and Barrett (־) في عام 1997 استخدام طريقة ترتيب النظام الوسطية Rank Order (Rentroit (ROC) Centroid method $\mathrm{W}_{\mathrm{i}(\mathrm{ROC})}=\left(\frac{1}{\mathrm{n}}\right) \sum_{\mathrm{j}=1}^{\mathrm{n}} 1 / \mathrm{j} \quad j=1$

حيث تمثل n عدد الفقرات و Wj الوزن للفقرة ال مئ

\section{ه- معايير الاداء الانشائي}

إن مقومات نجاح اداء أبي مشروع إنثائي هي الزمن والكلفة والنوعية والتي تمنل في مجموعها رؤوس منلث

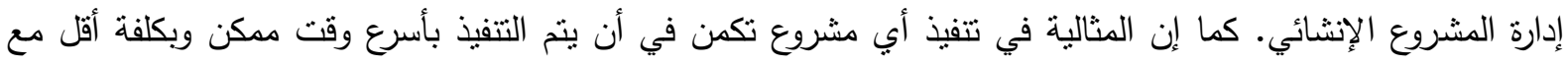
الحفاظ على النوعية المثبتة ضمن المواصفات الخاصة بذلك المشروع. 
1 - المفهوم العام

يقصد بالوقت في المشروع الإنشائي بانه "المدة الزمنية التي تم التخطيط والتعاقد عليها ليتم خلالها تتفيذ نشاطات

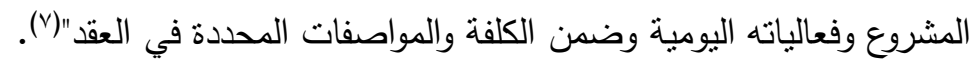

r - جدولة تنفيذ المشاريع

إن التخطيط في المشروعات الإنشائية هو عملية تسلسل منطقي وزمني لكيفية تتفيذ عناصر المشروع تستتد على

المعرفة والخبرة بأساليب التشييد ومواده وتطبيقاته(^).

r- طرائق جدولة (برمجة) المشاريع

توجد عدة طرائق لبرمجة زمن التتفيذ في مجال المشروعات الإنشائية، ومن هذه الطرق مايأني(ه) (9) (·'):

ب- طريقة منحني الكلفة (S-Curve Method)

| - -طريقة المخطط الثريطي

ج- طريقة التحليل الثبكي

ع - تقييم تقلم العمل في المشاريع

إن المبدأ الاساسي لعملية نقييم الوقت هو مقارنة المعلومات الفعلية لسير العمل في المشروع مع ما جاء في

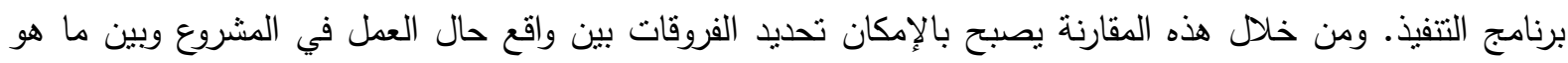

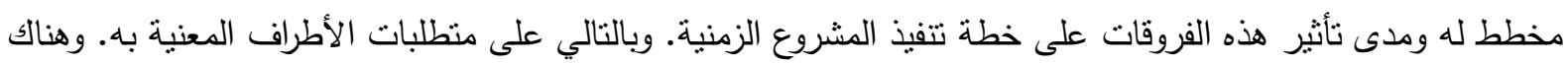

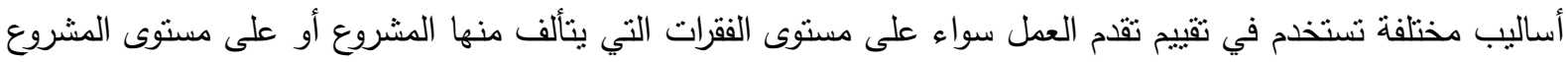
ككل أهمها('): ا- قياس وحدات العمل المنجزة للفقرة ب- النسبة المنقضية من المدة الزمنية المخصصة لتنفيذ الفقرة ت - الموارد المستخدمة في تتفيذ الفقرة ث- استخدام الخبرة في تقدير نسبة انجاز الفقرة ه- آلية التأخير في المشاريع الإنشائية

يتكون المشروع الإنشائي من مجموعه متصلة من الفعاليات. وإن الجدولة الفعلية لهذه الفعاليات قد تختلف عن

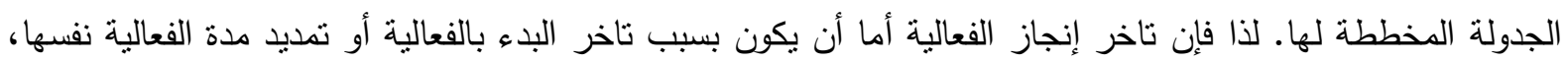

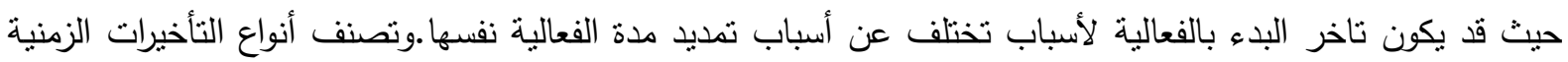

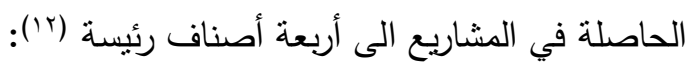

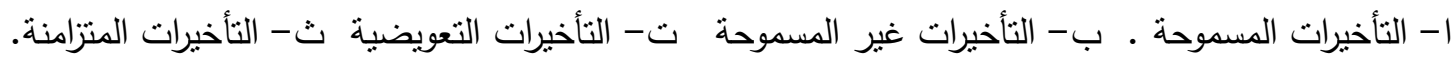
צ-الإخفاقات الزمنية أثناء تنفيذ المشاريع والعوامل المسببة لهات

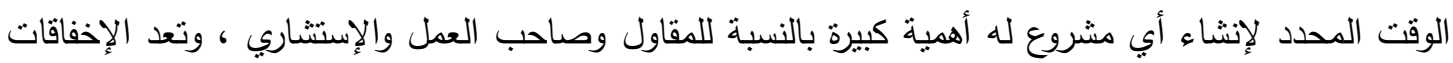
الزمنية من الامور التي لا يمكن ضمان عدم حصولها في المشروع الإنشائي. ففي بعض المشاريع لا نوجد سيطرة كافية

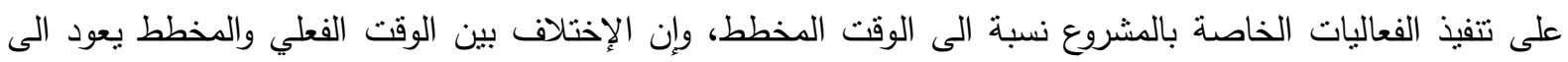
العديد من العوامل المسببة لهذه الإخفاقات.وهنالك مجموعة من الدراسات التي إهتمت بهذا المجال نتيجة لأهميته في تحديد مدى نجاح المشروع، ومن أهمها: دراسة أوجزت العوامل المؤثرة في حصول الإخفاقات الزمنية بالحالة الجويه، الفنشل في تجهيز العمالة الكافية،

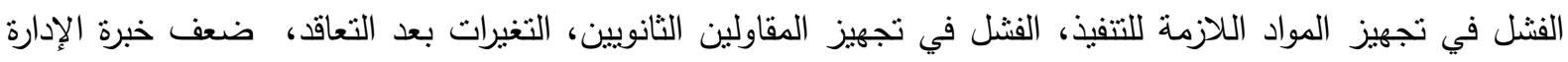

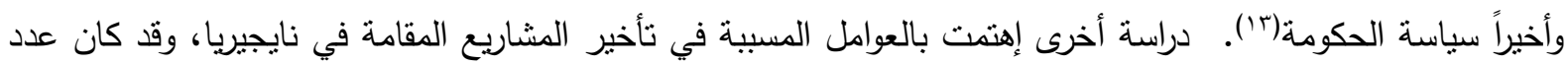

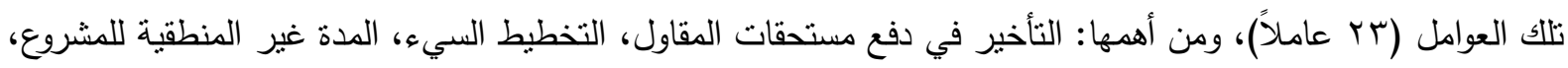
التغيرات في التصاميم وجداول الكميات، عدم الإلتزام بمحددات العقد، إدارة الإثراف غير كفوءة، سياسة الحكومة بالإضافة 
الى الأخطاء أثناء التتفيذ (؛) (). وإخرى، أوجزت نللك العوامل بعدم تكامل التصاميم والمواصفات، حدوث الأعاصير والهزات الأرضية، إضرابات العمال ومنازعاتهم والإخفاق في تجهيز المواد الإنشائية (10).

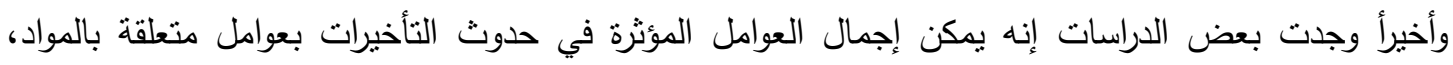

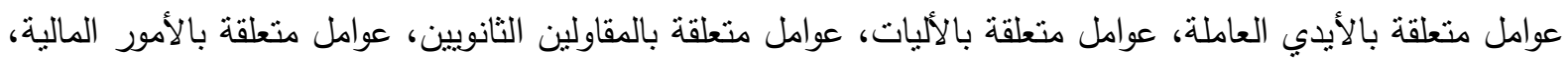

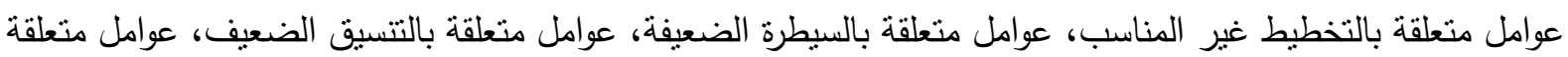
بطرائق التنفيذ غير المناسبة، عوامل متعلقة بالإثراف غير الكافي،عوامل متعلقة بالنقص في الكوادر الفنية، عوامل متعلقة

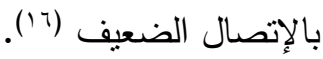

\section{ثاتيا:(الكلفة}

الكلفة بمفهومها العام تمنل المبالغ التي يتم صرفها عند شراء مواد أو لقاء خدمة ( كإجور الايدي العاملة، إجور معدات، مقاولات ثانوية او أية مصروفات أخرى). أما بالنسبة الى صاحب العمل فإن الكلفة تمثل المبالغ الكلية التي يتم

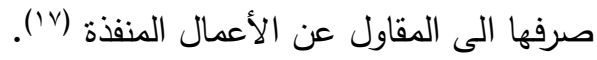
-

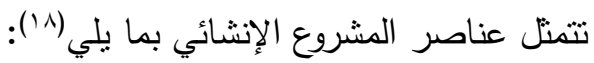
كلفة الأيدي العاملة ، كلفة المواد ، كلفة المكائن والمعدات ، كلفة المقاولات الثانوية ،كلفة التحميلات الإدارية .وبشكل عام، فإن كلفة أي مشروع إنشائي تتكون من نوعين أساسيين وهما الكلف المباشرة والكلف الغير مباشرة (9 (1). فالكلف الغير مباشرة هي الكلف التي يتحملها المشروع بأكمله في حين إن الكلف المباشرة هي الكلف الخاصة بكلف بكل فقرة

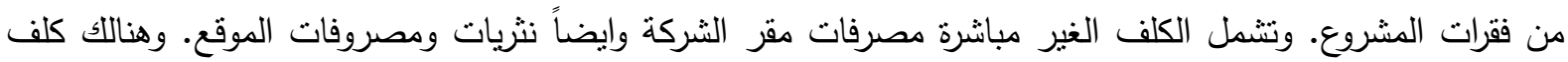
أخرى تتدرج ضمن الكلف الغير مباثرة كالفوائد على تمويل المشروع وكلفة الفرصة البديلة والغرامات التأخيرية التي قد وهن

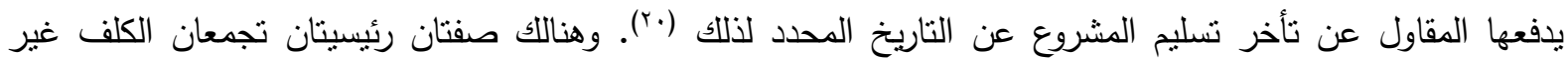

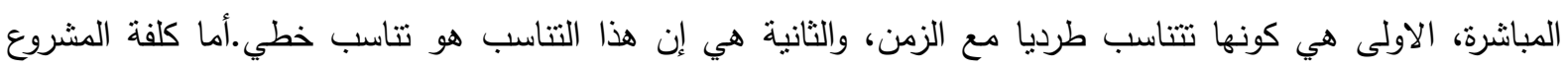

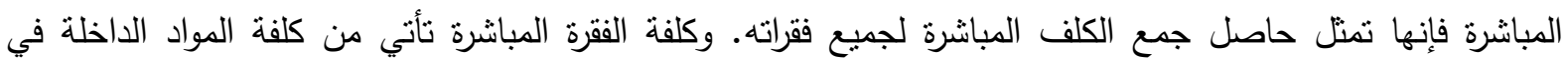
تتفيذها وكلفة الأيدي العاملة والمكائن والمعدات المطلوبة لتتفيذها بالإضافة الى كلف المقاولات الثانوية. وعلاقة الكلفة

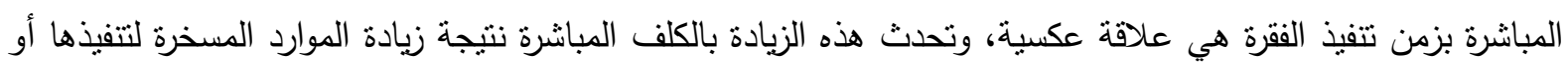
تغيير طرق الإنشاء المستخدمة لتنفيذها. r - العوامل المؤثرة على كلفة المشروع الإنشائي

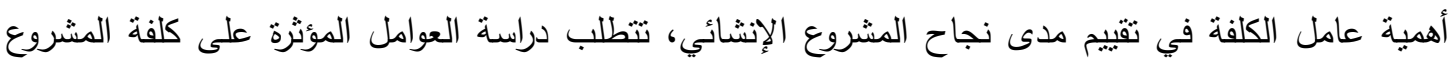
الانثائي، وتختلف هذه العوامل بإختلاف ظروف المشروع وظروف السوف السائدة، وقد أوجزت دراسات عديدة هذه

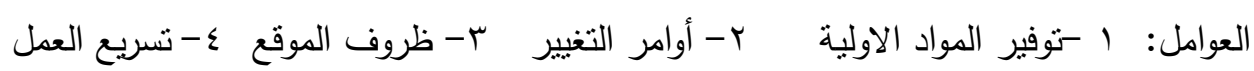
0- الوضع المالي للجهة المنفذة للمشروع وطبيعة القوانين.

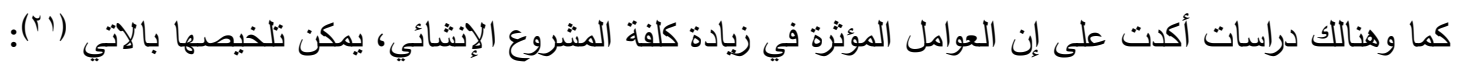
أ- التأخيرات التي تنجم عن الظروف الجوية، نوقفات وعطلات المكائن المفاجئة، نأخر المقاول الثنانوي وتأخر إيصسال المواد والمكائن والمعدات وتأثثرها في المباشرة أو الإستمرار بالعمل. 
ب- مصـروفات الأعمـال المعيبـة والمواد غير الصـالحة وإعـادة تتفيذها بصـورة ملائمسة والنفقات الإضـافية الناجمـة عن الإيقاف الفوري لحين قيام المقاول بإتخاذ الإجراءات التصحيحية التي ترضي المهند. ت - الأشراف والذي تتم من خلاله السيطرة على جميع موارد المشروع وعلى جميع عناصر التتفيذ. ث - سرعة التتفيذ الني قد نؤدي الى اللجوء الى ساعات إضافية، سرعة تهيئة المواد والمكائن. ج- التضخم والإرتفاع في الأسـعار حيث إن التضخم يعني نقصـان قيمة الوحدة النقدية مما يؤدي الى إرتفاع الأسعار فيمثل الكلف الغير متوقعة والتي لم يتم تضمينها في التخمينات الاصلية. ح- المخاطرة وعدم التأكد، حيث إن تقييم المخاطرة يعتبر عملية وقائية لضمان تقليل المفاجئات والنتائج السلبية التي تؤدي الى كلف متزايدة ومشروع متأخر .

\section{r- أسباب تغيير كلف المشاريع الإنشائية}

لغرض تحديد أسباب حصول التغييرات في الكلفة عند تقييم المشروع الإنشائي سواء كان هذا التقييم لأي فقرة من فقرات المشروع أو للمشروع ككل, فقد نم تحديد الجهات المسؤولة عن نشوء هذه التغييرات بالأطراف التي لها علاقة لأني بالمشروع (صاحب العمل في إدارة المشروع والإستشاري في إعداد مستتدات المشروع والمقاول في تتفيذ العمل، بالإضافة الى الظروف العامة المسؤولة عن أسباب خارج إرادة هذه الأطراف)، ومن ثم تحديد أسباب حصول التغينيرة إعبرات في الكلفة

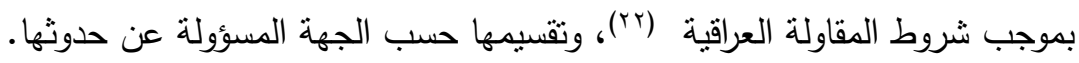
ع - السيطرة على الكلقة

يمكن تعريف السيطرة على الكلفة بانها جميع عمليات السيطرة على الانفاق في المشروع ابتداء من مرحلة ظهور

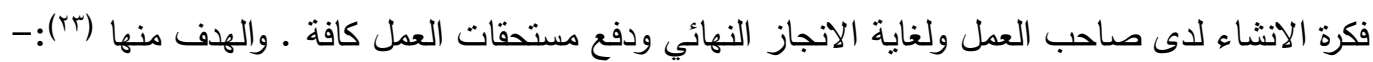

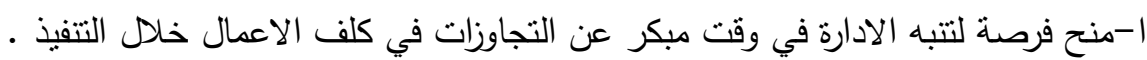
ب-تقييم مستوى انجاز الاعمال من حيث المدة والكلفة ومقارنتها مع مثيلاتها في الخطة.

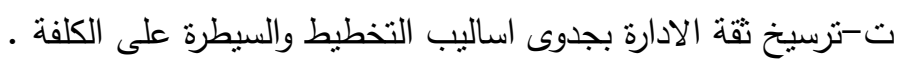
وهنالك بعض الادوات التخطيطية التي تساعد في عمليات التخطبط والسيطرة على الكلفة وتجعل منها عملية اكثر سهولة ودقة، هي: هيكل تجزئة العمل، جدول تحليل الكلفة، جدول الاسعار، جدول تحليل التنظيم، والترميز .وتوجد عدد من الانظمة الثائعة التي تنتخدم لغرض السيطرة على كلف المشاريع اثثاء تتفيذها، ويتضمن اي نظام سيطرة كلفوي على جلى عمليات لجمع وتوثيق البيانات الموقعية ومقارنة هذه البيانات مع ميزانيات محددة سابقا ومن ثم تحليل نتائج المقارنة

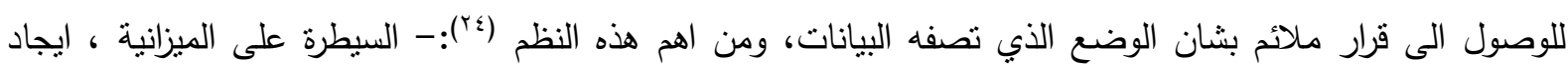

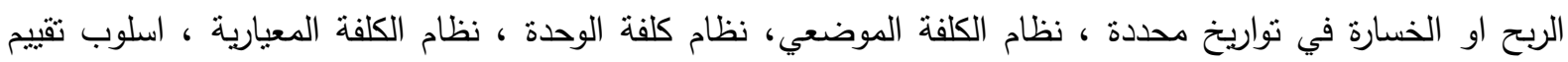
ومراجعة المشروع .وهنالك بعض الادوات الادارية التي تستطيع ان تقدم المعلومات ذات العلاقة بعملية السيطرة الكلفوية والتي يمكن استخدامها وتقديمها لمختلف المستويات الادارية، متخذة عدة اشكال او صور او جداول تساعد الادارة في عملية تحديد مواطن الخلل والضعف في تتفيذ فقرات المشروع، من اهمها:- منحنى الكلف، منحى القيم، منحنى الدخل النقدي، منحنى المتطلبات النقدية.

ثالثا: النوعية تعني النوعية"مطابقة التتفيذ للمنطلبات الفنية والتعاقدية للمشروع"(†)، ولهذا يجب أن نكون حجر زاوية في العملية الإنشائية من خلال إيجاد خطة محددة نوضح المستوى المطلوب من الجودة عن طريق تطبيق إجراءات تحكم 
الأطراف المعنية.إن النوعية تعرض عادة على شكل مواصفات ومخططات يجب أن تكون قابلة للتطبيق، منطلباتها واضحة كما إنها يجب ان تكون قابلة للقياس(بَ).

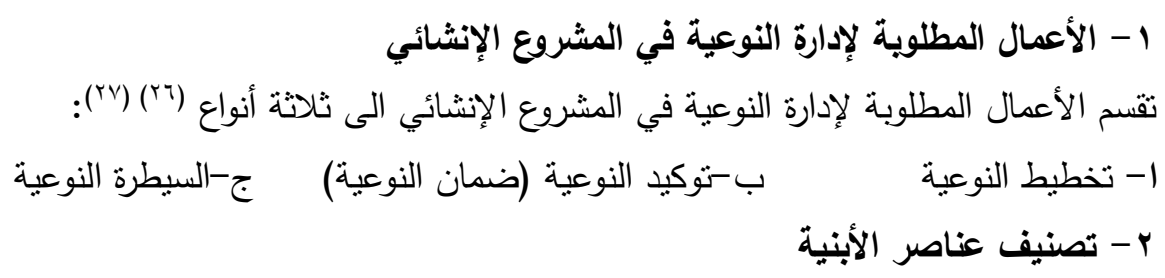

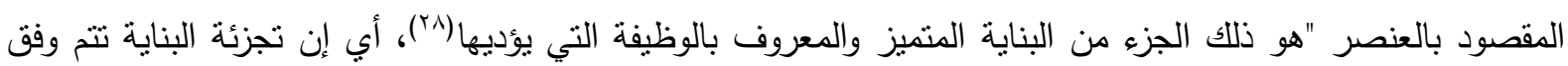
الأجزاء الوظيفية لها. ولغرض تحديد عدد العناصر التي تجزأ البناية اليها لا بد من إستخدام نظام محدد لتصنيف هذه بأه

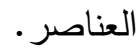

r- المخططات والمواصفات

ب-1 المخططات : مخططات التشييد هي رسومات تعرض الموقع والأبعاد وتفاصيل العمل المراد تتفيذه. وتشمل أنواع رسومات العقد، رسومات الموقع ورسومات العمل التفيلية. تتظم المخططات وترقم عادة طبقا للتخصص مثل إنثائية

وكهربائية وميكانيكية.

r-r المواصفات :تعرف على إنها هي تعليمات مكتوبة تكمل المخططات. وتحدد تحديداً كاملا شروط العمل ومنطلباته الفنية ونوع المواد ودرجة جودة المواد المصنعة التي يطلبها صاحب العمل وتعد معياراً ودليلاً للمقاول. وبإختصار فإن تُعان

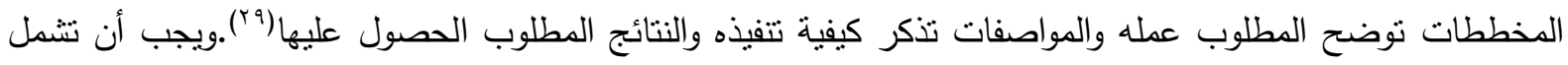
المواصفات على النصوص التي تحدد المقاييس وتبين النوعية وتوضح ما سيكون عليه المظهر النهائي حيث كلما كانت هذه العوامل واضحة سهلت عملية تقييم الفعالية أو المشروع ككل.

ع - الرقابة أو ضبط النوعية

هو تطبيق لخطة محكمة وإجراءات محددة تعد وتنفذ من قبل المقاول، يعتمدها ويتابع تطبيقها والتقييد بها إدارة الإتشراف في الموقع. الهذف من هذه الخطة مراقبة تتفيذ أعمال المشروع للتحكم بمستوى الجودة المطلوبة حسب وثائق العقد والمتطلبات الهندسية القياسية للأعمال التي ينفذها المقاول الرئيس أو المقاولون من الباطن، وذللك لتجنب أب خلل في التتفيذ أو تدن في الجودة قد تسبب إعادة التنفيذ وما يتزتب على ذلك من تأخبر وزيادة في النفقات وخلافه من سلسلة

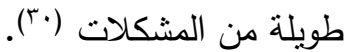

\section{ه-طرائق تأكيا وضبط النوعية}

هنالك عدة طرائق لضبط النوعية وتأكيدها في مشروعات البناء والنتشيبد الإنشائية، فقد يستخدم نوع واحد أو أكثر من نوع تبعا لطبيعة العنصر المراد ضبط وتأكبد جودته وظروف العمل ومستوى الجودة المطلوب، ومن هذه الطرائق:

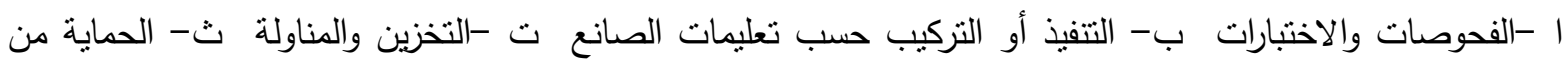

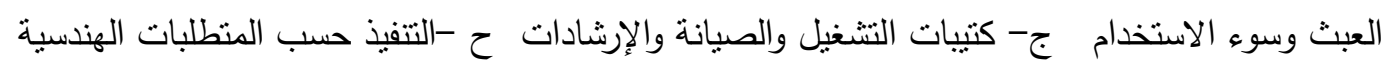

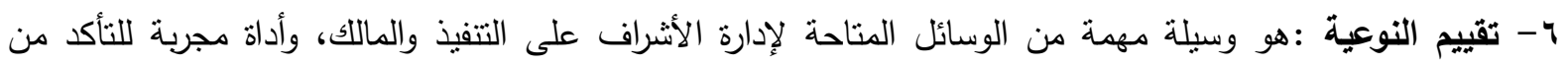

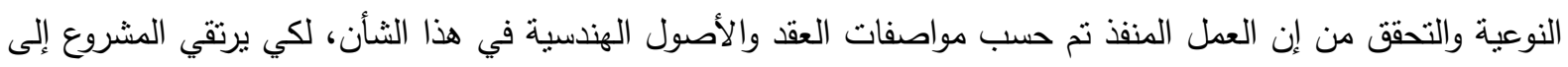
مستوى الجودة المطلوبة وليؤدي دوره الذي وجد من اجله كاملا كما حدده صاحب العمل. ينقسم التقييم الفني في مجال

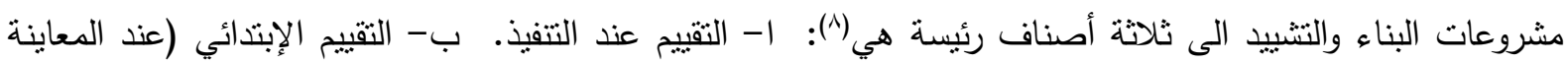
الأوليه بعد إنجاز المشروع). ت- التقييم النهائي (عند تسلّم المشروع من المقاول). 


\section{צ- تطبيق تقنية الـ COPRAS-G في عملية تقييم الاداء الانشائي للمشاريع}

باتباع خطوات عمل تقنية الـ COPRAS-G الموصوفة في المقاطع السابقة، يتمثل الهدف الاساس بنقييم اعمال

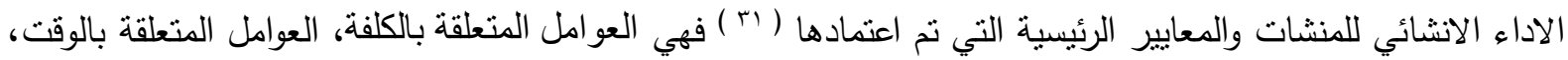
العوامل المتعلقة بالنوعية ، العوامل المتعلقة بالإنتاجية، العوامل المتعلقة بارضاء المالك، العوامل المتعلقة بإرضاء الانظمة والمجتمع، العوامل المتعلقة بالافراد، العوامل المتعلقة بالسلامة والامان، العوامل المتعلقة بالتجديد، العوامل المتعلقة بالبيئة.

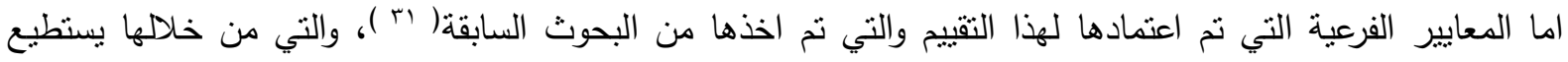
متخذوا القرارات ان يشيروا الى أفضليات كل بديل قرار في مصطلحات مساهنها في كل معيار،فهي مبينة في الجدول

وقد تم تقييم الاداء الانثائي لمشاريع محافظة ديالى عن طريق اجراء استبيان ميداني ينضمن المعايير المعتمدة

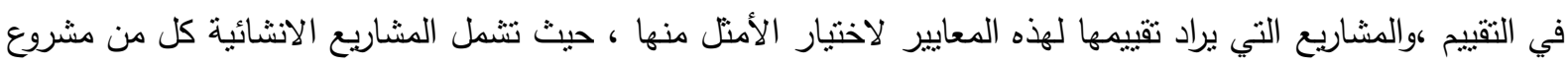
محطة مجاري بعقوبة، مشروع المستشفى الاسترالي، مشروع الاسكان الوطني - الغالبية ،مشروع الطريق الرئيسي بين تقاطع القس - المرادية

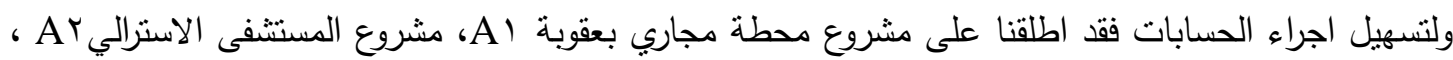

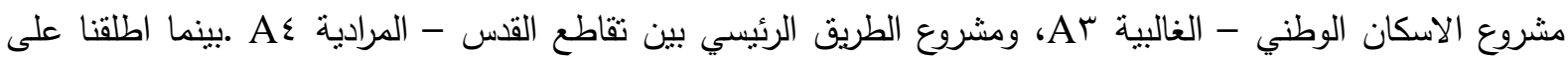

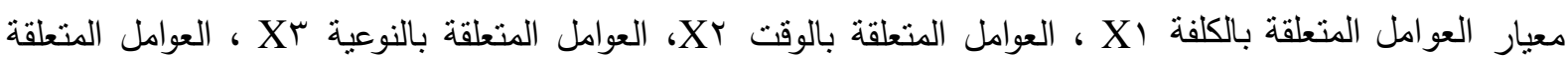

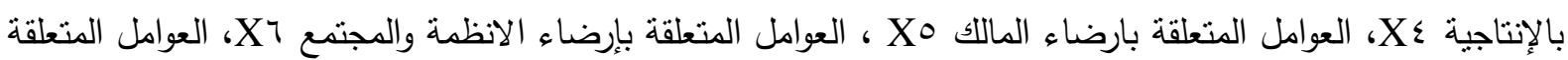

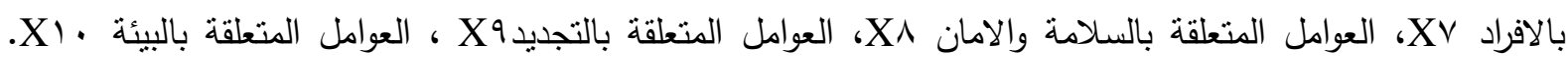

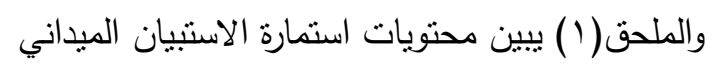

بعدها تم جدولة نتائج الاستبيان على ضوء الإجابات التي تم الحصول عليها، وكما مبين في الجداول (r) (r) (ع). ولغرض حساب الاهمية للمعايير من ناحية الاداء الانشائي للمشاريع ،و اعتمادا على ما ورد في خطوات تتفيذ طرق(مجموع الرتب، الترتيب المتبادل، ترتيب النظام الوسطية) في المقاطع السابقة ،تم اجراء حسابات الأهمية للمعايير في

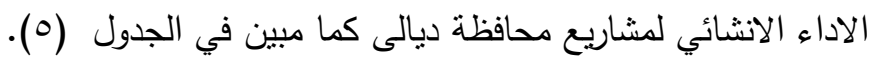
واعتمادا على ما ورد في خطوات تتفيذ تقنية COPRAS-G في المقطع (r)، نم اجراء الحسابات للمشاريع ضمن معايير الاداء الانشائي وكما مبين في الجدول (†).

\section{V - Vحليل ومناقشة نتائج الاستبيان - - V}

لقد تبين للباحث ومن خلال المقابلات المباشرة واجوبة الاستبيان النقاط التالية :1- لقد بينت المقابلات المباشرة ومن ثم طريقة ترنيب النظام الوسطية(ROC) ان معيار الكلفة هو اكثر اهمية من بقية

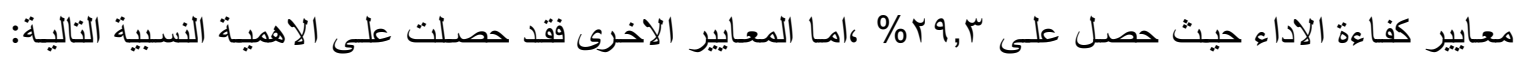

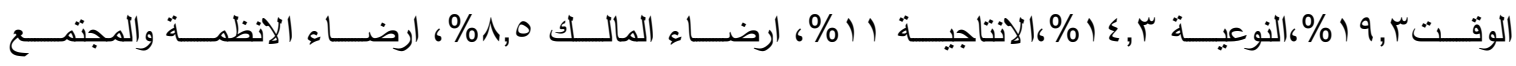

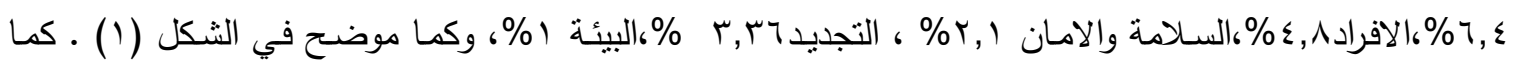
بينت المقابلات المباشرة ومن ثم طريقة مجموع الرتب(RS) ان معيار الكلفة هو اكثر اهية من بقية معايير كفاءة

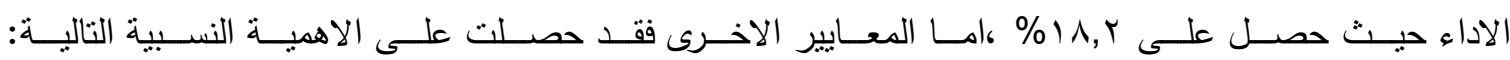




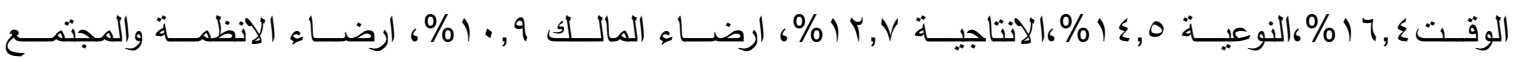

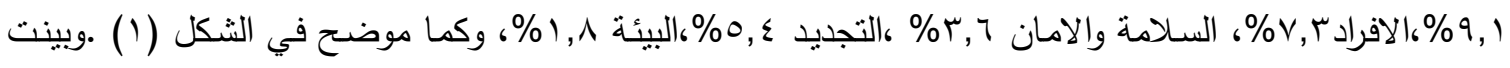
المقابلات المباشرة ومن ثم طريقة الترتيب المنبادل(RR) ان معيار الكلفة هو اكثر اهمية من بقية معايير كفاءة

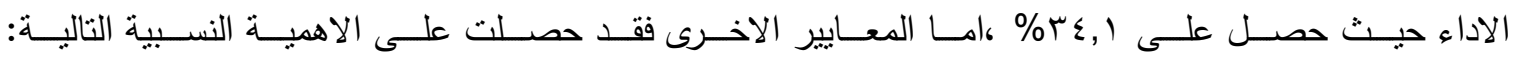

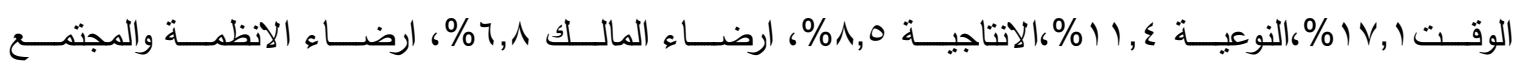

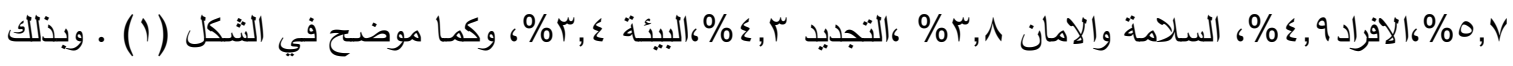

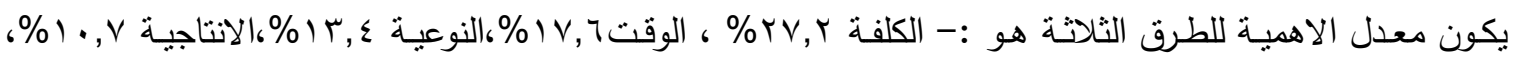

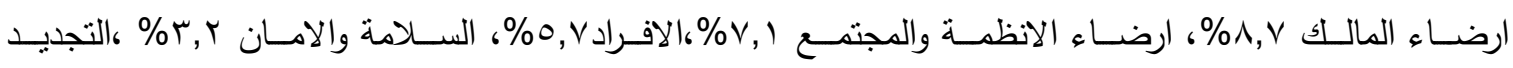
\% r, ) البيئة \% \% ץ- ان نتائج مقارنـة الاهمية للمشـاريع المشتمل عليها معياري الكلفة ، والوقت ، بينت ان معدل قيمـة الاهمية النسبية

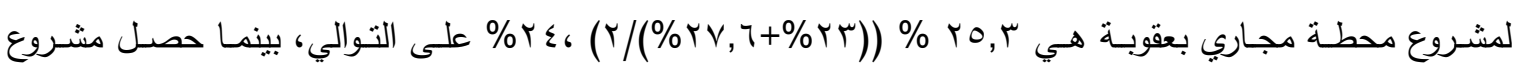

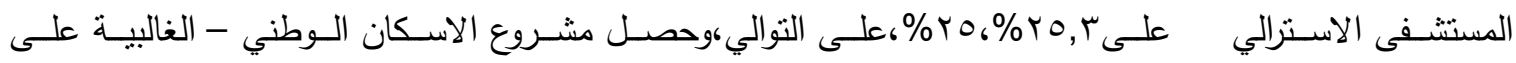

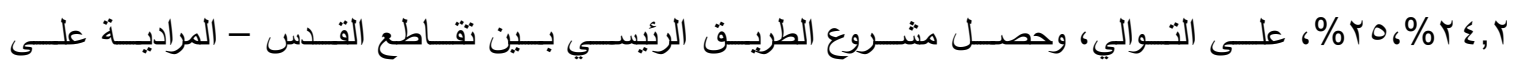

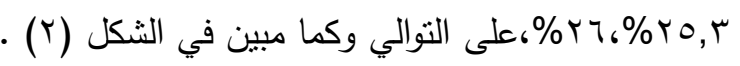
r- وبخصوص الاهمية النسبية لمعايير النوعية ،الإنتاجية،إرضـاء المالك ، حصل مشروع محطة مجاري بعقوبـة على

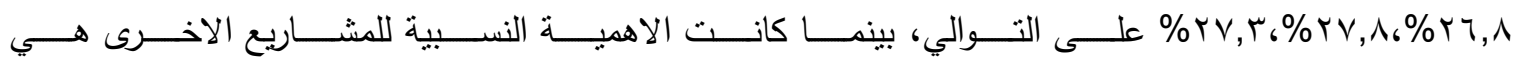

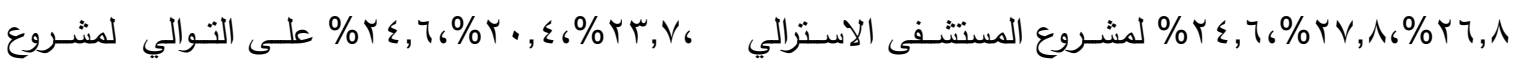

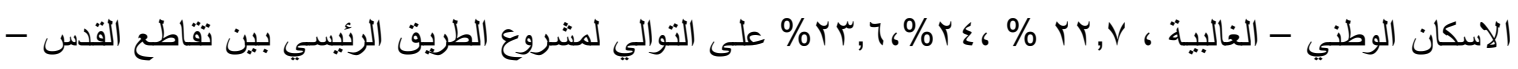

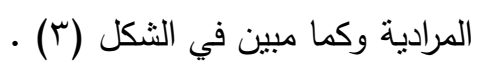
ع - واتضح من خلال اجابات افراد العينة ان مشروعي محطة مجاري بعقوبة و المسنتفى الاسترالي هي اكثر اهمية من بقية المشاريع فيما يخص معايير إرضاء الانظمة والمجتمع ، الافراد ، السلامة والامان، حيث حصل كل منهما على

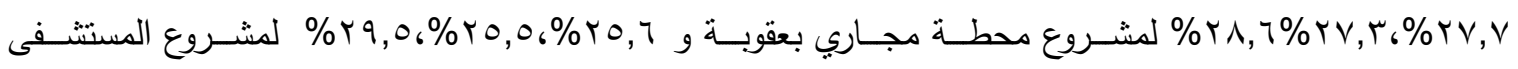
الاسترالي ، امـا المشـاريع الاخرى فقد حصلت على الاهمية النسبية التاليـة : مشروع الاسكان الوطني - الغالبيـة

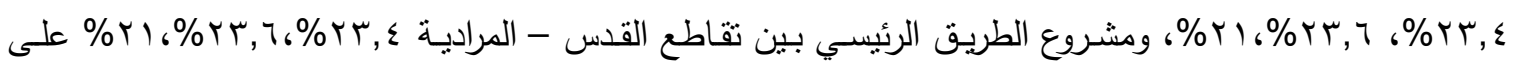

$$
\text { التوالي وكما مبين في الثكل ( ع ). }
$$

ه- ان نتائج مقارنـة الاهمية للمشـاريع المشتمل عليها معياري التجديد،والبيئة، بينت ان قيمـة الاهميـة النسبية لمشروع

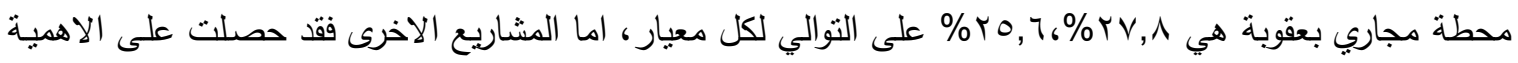

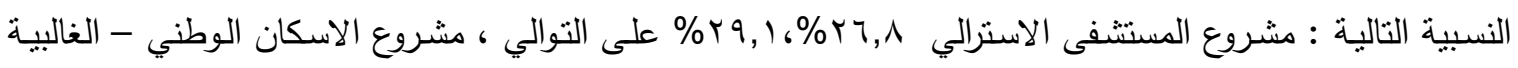

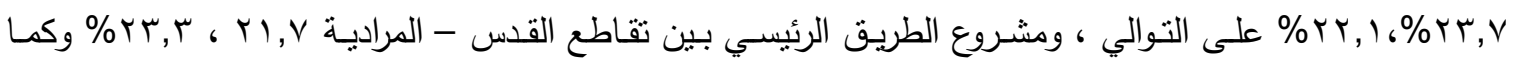

موضح في الثكل (0). (0) (1) 
من الاستتناجات التي تم التوصل اليها والتي نم استباطها من خلال البحث هي :-

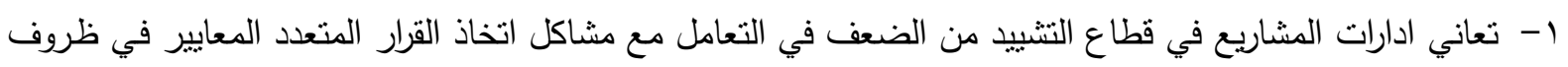
ضبابية

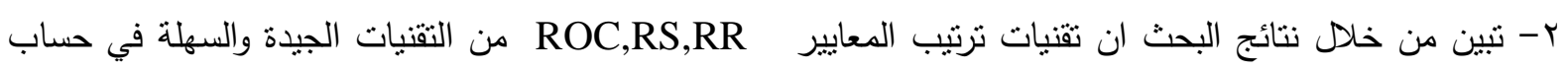

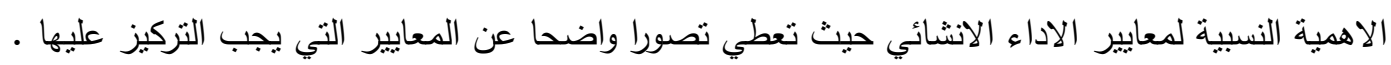

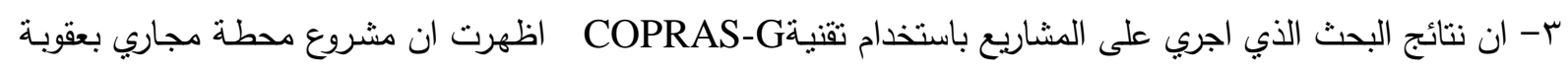

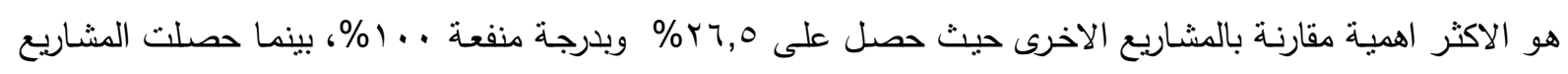

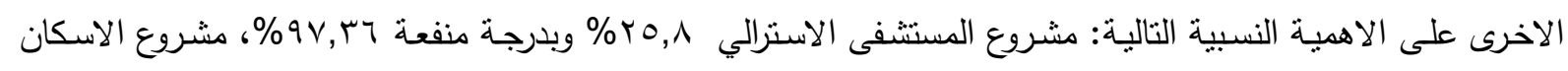

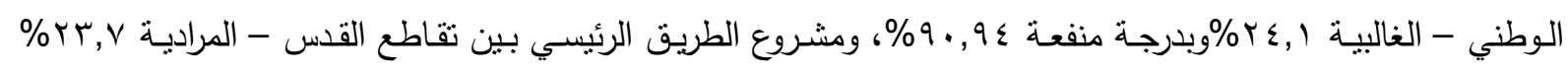

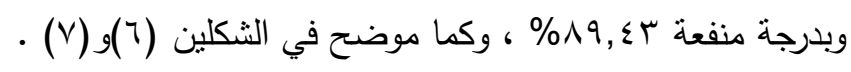
ع - تعاني ادارات المشاريع في قطاع التشييد في محافظة ديالى من عدم وجود اسلوب واضـح لتوثيق الاعمال المختصـة

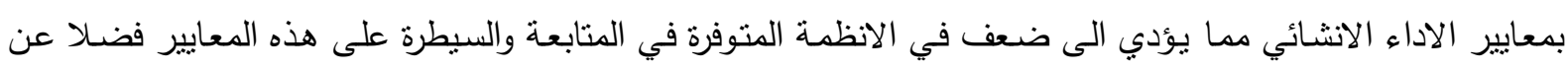

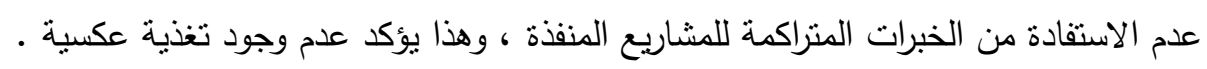

9

على ضوء الاستتاجات السابقة يمكن درج التوصيات التي تساهم في تحقيق اهداف البحث بشكل فعال ومؤثز ،وكما يلي 1- - ضرورة تطوير نقافة ادارة المشروع الصحيحة واستخدام تقنيات ادارة المشروع الكافية من خلال تعريف الكادر

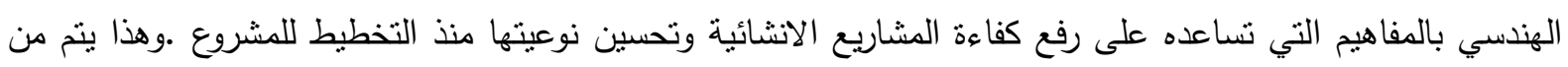
خال عمل دورات لتعريفهم بالاساليب الصحيحة و الحديثة للانثاء .

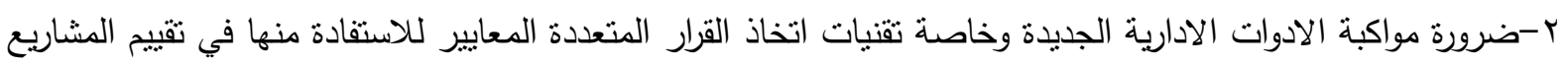
الجديدة ، من خلال تعريف الكادر الهندسي بهذه التقنية من خلال عمل دورات لتعريفهم بهذه التقنيات وكيفية نطبيقها وخاصة في ظروف من عدم التاكد واليقين. ץ- الاسراع في نطبيق النظم المقترحة في الاداء الانثائي بكافة فعاليتها من قبل الباحثين وذلك من خلاد عمل دورات تعريفية بهذه النظم واسلوب التعامل مع فعالياتها.

المصادر

'. Zavadskas, E.K., A. Kaklauskas, F. Peldschus and Z. Turskis. "Multi-Attribute DecisionMaking Model by Applying Grey Numbers", Vol. r•, No. r, r.o_r r., r . . .

r. Zavadskas, E.K., Kaklauskas,A.,Z. Turskis, Tamošaitien,J. "Selection of the effective dwelling house walls by applying attributes values determined at inttervals", vol. I $\varepsilon$, no. $r, r \cdot \Lambda$. 
تقييم الأداء الإنشائي لمشاريع محافظة ديالى باستخدام تقتية تقييم البدائل المتعددة المعايير النسبية المعقدة بعلاقات رمادية (COPRAS-G)

r. Deng, J. L. 19^ץ. “Control problems of grey system”, Systems and Control Letters '(०):

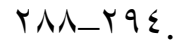

£. Deng, J. L. 19^^a. "Introduction to Grey System Theory", the Journal of Grey Theory ।: $1-r \leqslant$.

•. Stillwall, W.G., D.A.Seaver, and W.Edwards, "A Compaarsion of weight Approximation Techniques in Multiattribute Utility decision making", Organizational behavior and human performance, $r \wedge(1911), T r-V V$.

7. Barron, F. H. and Barrett, B. E. "Decision Quality Using Ranked Attribute Weights", Management Science, \&r (1997), 1010-10ro.

V. النحاس، ايهاب عبد الرزاق، "أثر إدارة الوقت على ضغوط العمل في الادارة العامة"، رسالة ماجستير في تخطيط القوى

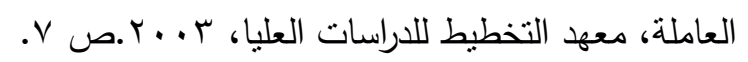

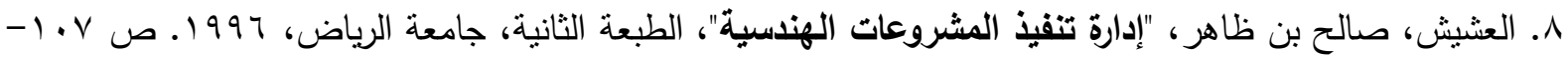

.rYO-Y1. 61. A 611 r

१. S. W., Nunnally, "Construction Method and Management", Published by, Prentice Hill, Upper Saddle River, New Jersey, 1991. Pages 01960Y.6011_09 .

1. Pratea Pusanond, Apirath, "A Comprehensive Practice of Total Float Pre-Allocation and Management for the Application of A CPM-Based Construction Contract", Thesis of Ph. D., Blacksburg, Virginia, Y..r. Page 17.

11. Clough, Richard H., "Construction Projects Management", John Wiley \& Sons, Inc., New York, I $9 \vee r$. Page $7 r, r \leqslant, ~ r \wedge$.

Ir. Diekmann, Krriaim Z. M., "Construction Delay in Construction Project", Journal of Construction Engineering and Management, Vol. 110, 1919. Page 091.

1r. Al-Khalil, Assaf \& Al-Hazim, "Causes of Delay in Large Building Construction Projects", ASCE, Vol. '1, 1990.page $\leqslant 0_{-} 0^{\circ}$.

1 £. Elimwa, Augustine Unch and Mangvwat Joshua, "Time-Overrun Factors in Nigerian

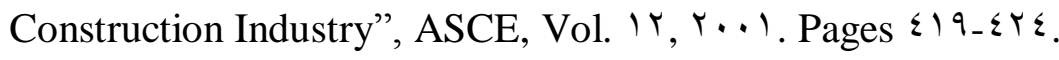

10. Chesley - Ayyers, P. E. "Specification for Architecture Engineering and Construction", rnd Edition, McGraw-Hill Book Company, New York, $19 \wedge r$. Page rтr.

17. Abdul Majed, M. ZMC, Caffer Ronald, "Factors of non-Excisable Delays that Influence Contractor Performance”, Journal of Management in engineering, Vol. । $₹, 199 \wedge$. Page కo.

IV. Merritt, Frederick S. \& Jonathan T. Ricketts, "Building Design and Construction Handbook", Six Edition, McGraw-Hill, r... . Page ro. 
1^. Rayburn, Letricia Gayle, “Cost Accounting Using a Cost Management Approach”, Sixth Edition, McGraw-Hill, USA, 1997.

19. Ostwald, Phillip F., "Construction Cost Analysis and Estimating”, Upper Saddle River, Prentice Hall, $\uparrow \ldots 1$. Page $\leqslant$.

• • † †لف، عبد السلام سر هيد، "دراسة مستوى الكلف غير المباشرة في مشاريع المباني"، رسالة ماجستير، قسم هندسة

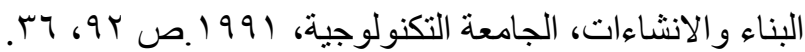

ץ. Gray, Clifford F. and Larson, Erik W., "Project Management: The Managerial Process",

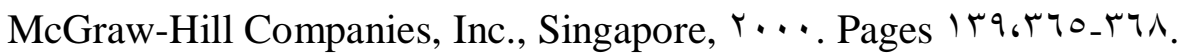

rr. Duncan, William R., "A Guida to the Project Management Body of Knowledge", Project Management Institute, USA, 1997.

rr. Miles, Derek and Paul Syagga, "Building Maintenance Management Manual", Intermediate Technology Publications, $19 \wedge \vee$.pages $r$,, ro, $r \wedge$.

$r \xi$. RPS \&INAC, "Project Management Manual", Technical Information Document, $r \cdots$. Page 7.

هץ . سمحيري، قيس زكي حنا، "دراسة تحليلية للكلف المتحققة لمشاريع المباني بالمقارنة مع كلف الاحالة"، رسالة

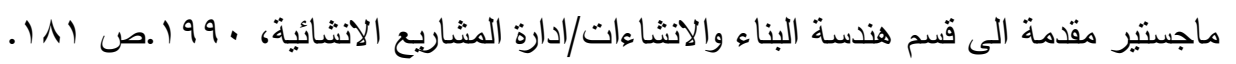

rч. Burns, James R., "Project and Process Management", All Rights Reserved Wide, r... T.pages $\left.r_{6} T_{6}\right) \cdot$ and 11 .

rv. Heizer, Jay and Render Barry, "Principles of Operations Management", rrd Edition, New Jersy, 1999. Page 149.

אץ. البناء، جرجيس، "تطبيقات على (SfB) في الصناعات الانثائية"، دورة انظمة المعلومات البنائية وتطبيقات على

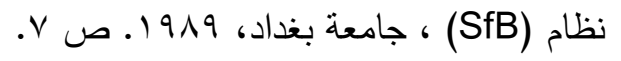

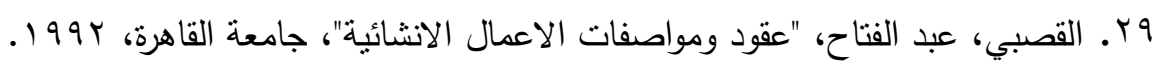

r. Hilton, Ronald W., "Cost Management", rnd Edition, McGraw-Hill Company, New

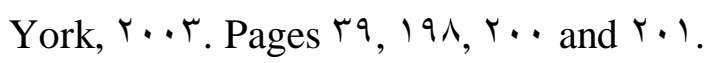

r. Saleh Samir Abu Shaban., "Factors Affecting the Performance of Construction Projects

in the Gaza Strip" A Thesis Submitted in Partial Fulfillment of the Requirements for Degree of Master of Science in Construction Management The Islamic University of Gaza. Palestine, April, ץ... 


\begin{tabular}{|c|c|c|}
\hline الاعتبارات & المعايير & $ت$ \\
\hline حجم المشاركة المالية في السوق للمؤسسة & \multirow{17}{*}{ العوامل المتعلقة } & \multirow[t]{17}{*}{1} \\
\hline السيولة النقدية للمؤسسة & & \\
\hline التدفق النقدي للمشروع & & \\
\hline 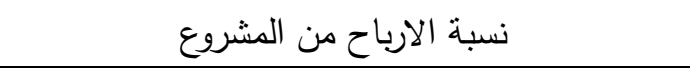 & & \\
\hline المصاريف الادارية للمشروع & & \\
\hline تكلفة التصميم للمشروع & & \\
\hline تكلفة المواد والمعدات للمشروع & & \\
\hline تكلفة العمالة للمشروع & & \\
\hline تكلفة ساعات العمل الاضافية للمشروع & & \\
\hline تكلفة الحوافز المادية & & \\
\hline تكلفة اعادة تتفيذ بعض الاعمال & & \\
\hline تكلفة الاوامر التغييرية & & \\
\hline نسبة الفاقد في المواد & & \\
\hline تحديث ميزانية المشروع بالنتظام & & \\
\hline نظام مراقبة التكاليف & & \\
\hline 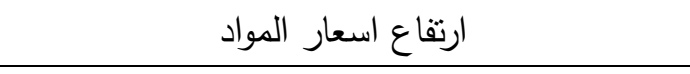 & & \\
\hline التغير في اسعار العملات & & \\
\hline الوقت اللازم لتجهيز الموقع & \multirow{9}{*}{ بالعوامل المتعلقة } & \multirow[t]{9}{*}{ r } \\
\hline المدة المقترحة لانشاء المشروع & & \\
\hline نسبة التاخر في الموافقة على اوامر العمل & & \\
\hline المدة اللازمة لتتفيذ الاوامر التغييرية & & \\
\hline المدة اللازمة لاصلاح او تعديل الاخطاء والعيوب & & \\
\hline معدل التاخير في الموافقة على المطالبات & & \\
\hline معدل التاخير في الدفعات المالية من المالك للمقاول & & \\
\hline توفر الموارد كما هو مخطط له وحسب مدة المشروع & & \\
\hline معدل التاخير بسبب اغلاق الطرق وقلة المواد & & \\
\hline مدى الالتزام بالمواصفات والثروط المتقق عليها & \multirow{6}{*}{ بالنوامل المتعلقة } & \multirow[t]{6}{*}{$r$} \\
\hline وجود الاشخاص ذوي الكفاءة والخبرة العالية & & \\
\hline جودة المواد الخام والمعدات المستخدمة في المشروع & & \\
\hline مشاركة المستويات الادارية للشركة في اتخاذ القرارات & & \\
\hline وجود نظام لتقييم الجودة في المؤسسة & & \\
\hline وجود اجتماعات ودورات تدريبية متعلقة بالجودة & & \\
\hline
\end{tabular}




\begin{tabular}{|c|c|c|}
\hline مدى التعقيد الموجود في المشروع & \multirow{5}{*}{ العوامل المتعلقة } & \multirow[t]{5}{*}{$\varepsilon$} \\
\hline عدد المشاريع الجديدة في السنة & & \\
\hline العلاقة بين العمال وادارة المشروع & & \\
\hline معدل غياب العاملين في المشروع & & \\
\hline تتابع انشطة المشروع حسب الجدول الزمني & & \\
\hline التتسيق في تبادل المعلومات بين المالك وطاقم المشروع & \multirow{5}{*}{ العوامل المتعلقة } & \multirow[t]{5}{*}{ 。 } \\
\hline المهارات القيادية لمدير المشروع & & \\
\hline السرعة والكفاءة في تقديم الخدمة للمالك & & \\
\hline عدد الخلافات والنزاعات بين المالك وطاقم المشروع & & \\
\hline عدد الاعمال المطلوب اعادتها & & \\
\hline التكلفة اللازمة للالتزام بالانظمة & \multirow{4}{*}{ بإرضاء الانظملة المتعلقة } & \multirow[t]{4}{*}{7} \\
\hline عدد الاعمال المخالفة للانظمة & & \\
\hline جودة وتوفر الاوراق والمستتدات الرسمية والنظامية & & \\
\hline المشاكل الناتجة عن الجيران والظروف المحيطة بالموقع & & \\
\hline سلوك الموظفين في المشروع & \multirow[t]{4}{*}{ العوامل المتعلقة بالافراد } & \multirow[t]{4}{*}{$\checkmark$} \\
\hline تعزيز روح المنافسة بين الموظفين & & \\
\hline تحفيز الموظفين & & \\
\hline الانتماء للعمل (مكان المشروع وموقعه) & & \\
\hline مدى تطبيق عوامل الامان والسلامة في المشروع & \multirow{4}{*}{ بالسلامة والامل المتعلقة } & \multirow[t]{4}{*}{$\Lambda$} \\
\hline سهولة الوصول الى الموقع (مكان المشروع وموقعه) & & \\
\hline نسبة الحوادث المسجلة في المشروع & & \\
\hline نسبة التعويضات الناتجة عن الحوادث للعاملين وغيرهم & & \\
\hline التعلم من الخبرة الذاتية ومن الخبرة السابقة & \multirow{5}{*}{ العوامل المتعلقة } & \multirow[t]{5}{*}{9} \\
\hline التعلم من الاداء الافضل والخبرات لدى الاخرين & & \\
\hline تدريب الموارد البشرية بالمهارات الجديدة واللازمة للمشروع & & \\
\hline العمل الجماعي & & \\
\hline مراجعة الاخطاء والمشاكل ووضع الحلول المناسبة لها & & \\
\hline 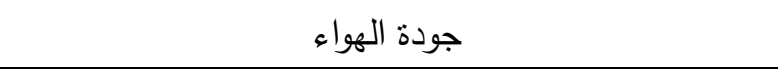 & \multirow[t]{4}{*}{ العوامل المتعلقة بالبيئة } & \multirow[t]{4}{*}{1.} \\
\hline 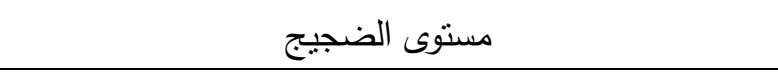 & & \\
\hline النفايات الموجودة حول الموقع & & \\
\hline الظروف المناخية في الموقع & & \\
\hline
\end{tabular}




\begin{tabular}{|c|c|c|c|c|c|c|c|c|c|}
\hline & & \multicolumn{8}{|c|}{ 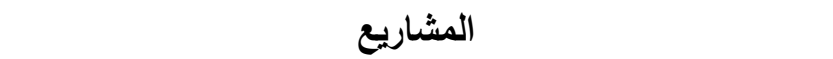 } \\
\hline & & \multicolumn{2}{|c|}{ مشروعا } & \multicolumn{2}{|c|}{ 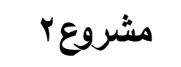 } & \multicolumn{2}{|c|}{ مشروعr } & \multicolumn{2}{|c|}{ 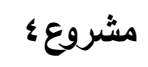 } \\
\hline & & $\mathbf{L}$ & $\mathbf{U}$ & $\mathbf{L}$ & $\mathbf{U}$ & $\mathbf{L}$ & $\mathbf{U}$ & $\mathbf{L}$ & $\mathbf{U}$ \\
\hline \multirow{10}{*}{$\overline{3}$} & العوامل المتعلقة بالكلقة & ๑. & 9. & 0 . & 7. & $\leqslant 0$ & 9. & o. & 9. \\
\hline & العوامل المتعلقة بالوقت & 00 & 7. & 00 & 70 & 00 & 90 & 7. & 90 \\
\hline & العوامل المتعلقة بالجودة & 9. & v. & 9. & v. & ○. & 90 & o. & 9. \\
\hline & العوامل المتعلقة بالإنتاجية & v. & $\wedge$. & $v$. & $\wedge$. & ๑. & 7. & 9. & v. \\
\hline & العوامل المتعلقة بارضاء المالك & v. & $\wedge$. & 9. & Vo & 7. & vo & 7. & v. \\
\hline & العوامل المتعلقة باررضاء الانظمة & 9. & v. & 7. & $v$. & ๑. & 7. & o. & 7. \\
\hline & العوامل المتعلقة بالافراد & v. & $\wedge$. & $v$. & $\wedge$. & 7. & v. & 7. & v. \\
\hline & العوامل المتعلقة بالسلامة & v. & $\wedge$. & $v$. & 10 & o. & 7. & 0 . & 7. \\
\hline & العوامل المتعلقة بالتجديد & 7. & v. & 7. & vo & 0 . & 70 & $\leqslant 0$ & 7. \\
\hline & العوامل المتعلقة بالبيئة & 9. & v. & 7. & vo & $\varepsilon$. & 00 & $\varepsilon$. & 7. \\
\hline
\end{tabular}

\begin{tabular}{|c|c|c|c|c|c|c|c|c|c|c|c|}
\hline & & \multicolumn{8}{|c|}{ المشاريع } & \multirow{2}{*}{\multicolumn{2}{|c|}{ المجموع }} \\
\hline & & \multicolumn{2}{|c|}{$A^{\prime}$} & \multicolumn{2}{|c|}{$\mathbf{A}^{r}$} & \multicolumn{2}{|c|}{$A^{r}$} & \multicolumn{2}{|c|}{$A^{2}$} & & \\
\hline \multirow{11}{*}{ 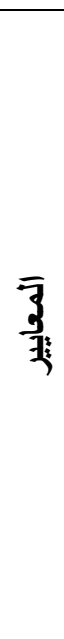 } & & $\mathbf{L}$ & $\mathbf{U}$ & $\mathbf{L}$ & $\mathbf{U}$ & $\mathbf{L}$ & $\mathbf{U}$ & $\mathbf{L}$ & $\mathbf{U}$ & & \\
\hline & $\mathbf{X}^{\prime}$ & 0. & 7. & 0 . & 7. & $\leqslant 0$ & 7. & 0. & 7. & 190 & $r \leq$. \\
\hline & $\mathbf{X}^{r}$ & 00 & 7. & 00 & 70 & 00 & 70 & 9. & 70 & YYO & $r 00$ \\
\hline & $\mathbf{X}^{\mu}$ & 7. & $\mathrm{~V}$. & 9. & $V_{\text {. }}$ & 0. & 70 & 0. & 7. & Yr. & Y70 \\
\hline & $X^{\varepsilon}$ & $\mathrm{V}$. & $\Lambda$. & $V$. & $\wedge$. & 0. & 7. & 7. & V. & ro. & rq. \\
\hline & $\mathrm{X}^{0}$ & $V$. & $\Lambda$. & 7. & Vo & 7. & Vo & 7. & $V$. & ro. & $r \ldots$ \\
\hline & $X^{7}$ & 7. & $V$. & 00 & 70 & 0. & 7. & 0. & 9. & Ylo & $r \otimes 0$ \\
\hline & $\mathbf{X}^{\mathrm{V}}$ & $\mathrm{V}$. & $\Lambda$. & 70 & Vo & 9. & $V$. & 9. & $V$. & ros & $r 90$ \\
\hline & $\mathbf{X}^{\wedge}$ & $V$. & $\Lambda$. & $V$. & $\wedge 0$ & 0. & 7. & 0. & 7. & $r \leq \cdot$ & $r \wedge \theta$ \\
\hline & $\mathrm{X}^{9}$ & 7. & Vo & 7. & $V$. & 0. & 70 & $\leqslant 0$ & 9. & Ylo & $r V$. \\
\hline & $X^{\prime}$ & 0. & 7. & 00 & $V \cdot$ & $\varepsilon$ & 00 & $\varepsilon$ & 9. & 110 & $r \leqslant 0$ \\
\hline
\end{tabular}


جدول رقم (ء): مصفوفة قرار التطبيع لمعايير تقييم الأداء الإنشائي للمشاريع.

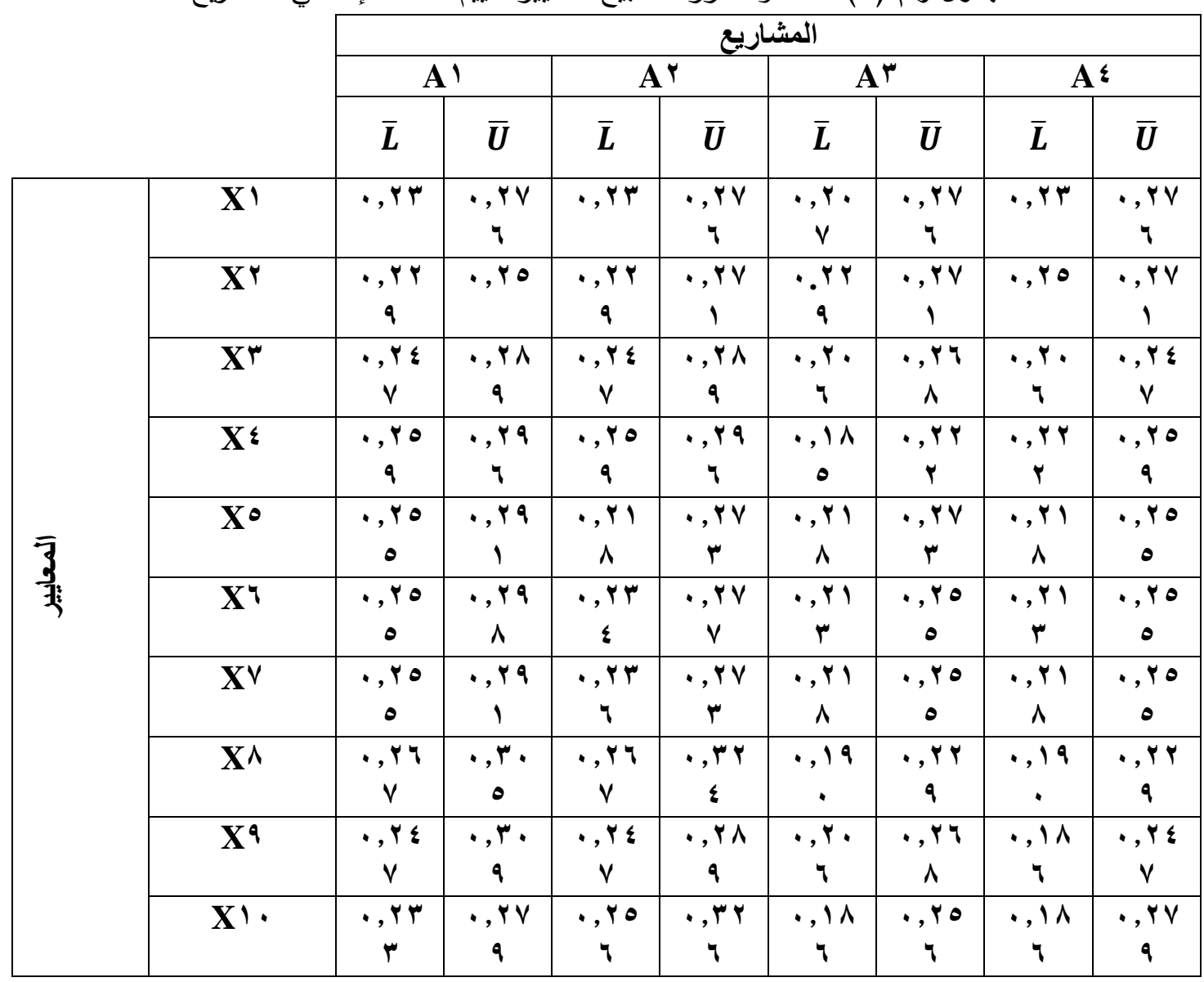

جدول رقم (0): حسابات الأهمية لمعايير الأداء الإنشائي.

\begin{tabular}{|c|c|c|c|c|c|c|c|c|}
\hline المعيار & $\begin{array}{l}\text { ترتيب } \\
\text { (m) }\end{array}$ & (المعيرتيب & $W_{i(\text { ROC })}$ & (ترجموع & $W i(R S)$ & (المعيار) & $W i(R R)$ & الاوزان \\
\hline$\overline{X^{\prime}}$ & 1 & r,q Yo & •,Yqr & 00 & $1 \wedge r$ & $r, q r q$ & $\cdot, r \leq 1$ & 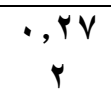 \\
\hline $\mathbf{X}^{r}$ & $r$ & 1,9Yo & $\cdot, 194$ & 00 & $\cdot, 17 \varepsilon$ & $r, q r q$ & $\cdot,|v|$ & $\cdot \frac{, I V}{7}$ \\
\hline $\mathbf{X}^{r}$ & $r$ & $1, \leqslant Y 0$ & $\cdot, 1 \leq r$ & 00 & $\cdot, 1 \leq 0$ & r.q & $\cdot, 11 \leq$ & $\begin{array}{c}\cdot, 1 r \\
\varepsilon\end{array}$ \\
\hline$X^{2}$ & $\varepsilon$ & $1, .90$ & $\cdot, 11$ & 00 & $\cdot, I Y V$ & Y,qYq & $\cdot, \wedge \theta$ & $\stackrel{\cdot 1}{v}$ \\
\hline $\mathrm{X}^{0}$ & 0 & $\cdot, \wedge \leq \nearrow$ & $\cdot, \cdot \wedge \theta$ & 00 & $\cdot, 1 \cdot 9$ & $r, q r q$ & $\cdot, \cdot 91$ & $\cdot{ }_{V} \cdot \wedge$ \\
\hline$X^{7}$ & 7 & $\cdot, 7 \leq 7$ & $\cdot,, 7 \varepsilon$ & 00 &., .91 & $r, q r q$ &., $.0 \mathrm{~V}$ & $\begin{array}{c}\cdot, \cdot v \\
1\end{array}$ \\
\hline $\mathbf{X}^{\vee}$ & $\mathrm{V}$ & $\cdot, \leqslant \vee q$ & $\cdot, \cdot \leqslant \wedge$ & 00 & $\cdot, \cdot V r$ & $r, q r q$ & $\cdot, \cdot \leq q$ & $\stackrel{\cdot, 0}{v}$ \\
\hline $\mathbf{X}^{\wedge}$ & 9 & $\cdot, Y \backslash 1$ & $\cdot, \cdot Y I$ & 00 & •, . M & $r, q r q$ & $\cdot, \cdot r \Lambda$ & $\cdot, \cdot r$ \\
\hline
\end{tabular}


تقييم الأداء الإنشائي لمشاريع محافظة ديالى باستخدام تقتية تقييم البدائل المتعددة المعايير النسبية المعقدة بعلاقات رمادية (COPRAS-G)

\begin{tabular}{|c|c|c|c|c|c|c|c|c|}
\hline & & & & & & & & $r$ \\
\hline $\mathrm{X}^{9}$ & $\Lambda$ & צr, & $\cdot, \cdot r r$ & 00 &., $0 \leqslant$ & r,q $q q$ & $\cdot, \cdot \leq r$ & • \\
\hline $\mathbf{X}^{1}$. & 1. & $\cdot, 1$ & $\cdot, \cdot 1$ & 00 & $\cdot, \cdot 11$ & $r, q$ rq & צ & $\begin{array}{c}\cdot, r \\
1\end{array}$ \\
\hline
\end{tabular}

جدول رقم(†): حسابات مؤشرات التعظيم والتقليل وأوليات البدائل ودرجة المنفعة لها.

\begin{tabular}{|c|c|c|c|c|c|c|c|c|c|c|c|}
\hline & \multirow[t]{3}{*}{ * } & \multirow[t]{3}{*}{ الوزن } & \multicolumn{8}{|c|}{ المشاريع } \\
\hline & & & & \multicolumn{2}{|c|}{$\mathbf{A}^{\prime}$} & \multicolumn{2}{|c|}{$A^{r}$} & \multicolumn{2}{|c|}{$\mathbf{A}^{r}$} & \multicolumn{2}{|c|}{$A^{\varepsilon}$} \\
\hline & & & & $\hat{\mathbf{L}}$ & $\widehat{\mathbf{U}}$ & $\hat{\mathbf{L}}$ & $\widehat{\mathbf{U}}$ & $\hat{\mathbf{L}}$ & $\widehat{\mathbf{U}}$ & $\hat{\mathbf{L}}$ & $\widehat{\mathbf{U}}$ \\
\hline \multirow{10}{*}{$\overline{\overline{3}}$} & $\mathbf{X}^{\prime}$ & - & $\cdot, Y V Y$ & י , , & $\cdot, \cdot \vee v$ & .,.74 & $\cdot, \cdot \vee v$ &., .07 & $\begin{array}{c}\cdot, \cdot V \\
0\end{array}$ & $\cdot, \cdot 7 r$ & $\cdot \stackrel{\cdot \cdot V}{0}$ \\
\hline & $\mathbf{X}^{r}$ & - & $\cdot, 1 \vee 7$ & $\cdot, \cdot \leq$ & $\cdot, \cdot \leq \leqslant$ & $\cdot, \cdot \varepsilon$ & $\cdot, \cdot \leqslant \Lambda$ & $\cdot, \cdot \varepsilon$ & $\begin{array}{c}\cdot, \cdot \varepsilon \\
\wedge\end{array}$ & $\cdot, \cdot \leq \leqslant$ & $\begin{array}{c}\cdot, \cdot \varepsilon \\
\wedge\end{array}$ \\
\hline & $\mathbf{X}^{\mu}$ & + & $\cdot, I T \varepsilon$ & $\cdot, \cdot r r$ & $\cdot, \cdot r q$ & $\cdot, \cdot T r$ & $\cdot, \cdot r q$ & $\cdot, \cdot Y \wedge$ & $\cdot \frac{,+r}{4}$ & $\cdot, \cdot Y \wedge$ & $\cdot \stackrel{r}{r}$ \\
\hline & $X^{\varepsilon}$ & + & $\cdot, 1 \cdot v$ & $\cdot, \cdot Y \wedge$ & $\cdot, \cdot M r$ & $\cdot, \cdot Y \Lambda$ & , , Mr & $\cdot, \cdot r$ & $\begin{array}{c}\cdot,+r \\
\varepsilon\end{array}$ & $\cdot, \cdot Y \leq$ & $\begin{aligned} \cdot, \cdot r \\
\Lambda\end{aligned}$ \\
\hline & $\mathrm{X}^{0}$ & + & $\cdot, \wedge \vee$ & $\cdot, \cdot Y r$ & $\cdot, \cdot Y O$ & $\cdot, .19$ & $\cdot, \cdot Y \leq$ & $\cdot, .19$ & $\stackrel{\cdot, r}{\varepsilon}$ & $\cdot, .19$ & $\cdot \vec{r}$ \\
\hline & $\mathrm{X}^{7}$ & + & $\cdot, \cdot V 1$ & $\cdot, \cdot 11$ & $\cdot, \cdot Y I$ & $\cdot, \cdot 1 \mathrm{~V}$ & $\cdot, \cdot r$ & $\cdot, \cdot 10$ & $\begin{array}{c}\cdot,+1 \\
\wedge\end{array}$ &., .10 & $\begin{array}{c}\cdot,+1 \\
\wedge\end{array}$ \\
\hline & $\mathbf{X}^{\mathrm{V}}$ & + & $\cdot, . \otimes V$ &.,+10 & $\cdot, \cdot 1 \mathrm{~V}$ & $\cdot, \cdot 1 r$ & $\cdot, .17$ & $\cdot, \cdot I r$ & $\cdot \stackrel{+1}{0}$ & $\cdot, \cdot I r$ & $\cdot, \cdot 1$ \\
\hline & $\mathbf{X}^{\wedge}$ & + & $\cdot, \cdot M r$ & $9, \cdots 9$ & $\cdot, \cdot 1$ & $\cdot, \cdots 9$ & $\cdot, \cdot 1$ & $\cdot, \cdots 9$ & $\ddot{v}, \cdot$ & $\cdot, \cdots 9$ & $\ddot{v}$ \\
\hline & $\mathrm{X}^{9}$ & + & $\cdot, \cdot \varepsilon r$ & $\cdot, \cdot 11$ & $\cdot, \cdot 1 r$ & $\cdot,+11$ & $\because \cdot I r$ & $\cdot, \ldots 9$ & $\ddot{r}, \cdot 1$ & $\cdots, \cdots$ & $\cdot,+1$ \\
\hline & $\mathbf{X}^{\prime}$ & + & $\cdot, \cdot Y I$ & 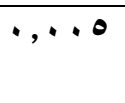 & $\cdot, \cdots 7$ & $\cdot, \cdots 0$ & $\cdot, \cdots V$ & $\cdot, \cdots \varepsilon$ & $\ddot{\circ}$ & $\cdot, \cdots \varepsilon$ & 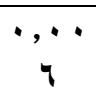 \\
\hline \multicolumn{4}{|c|}{ 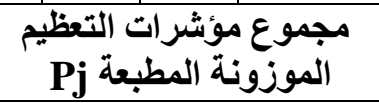 } & \multicolumn{2}{|c|}{$\cdot, 10 Y$} & \multicolumn{2}{|c|}{$\cdot, 1 \leq V$} & \multicolumn{2}{|c|}{$\cdot, I Y V$} & \multicolumn{2}{|c|}{$\cdot, I Y \wedge$} \\
\hline \multirow{2}{*}{\multicolumn{4}{|c|}{ مجموع مؤشرات التقليل الموزونة المطبعة Rj }} & \multicolumn{2}{|c|}{., 111} & \multicolumn{2}{|c|}{., 114} & \multicolumn{2}{|c|}{., 11} & \multicolumn{2}{|c|}{., 110} \\
\hline & & & & \multicolumn{4}{|c|}{$\sum R j=0.449$} & \multicolumn{3}{|c|}{$\sum \frac{1}{R j}=35.65$} & \\
\hline \multicolumn{4}{|c|}{ Qالأهمية النسبية للببليل } & \multirow{2}{*}{\multicolumn{2}{|c|}{$\frac{., Y 70}{\% 1 \cdots}$}} & \multicolumn{2}{|c|}{$\cdot, r \otimes \Lambda$} & \multicolumn{2}{|c|}{$\cdot, Y \leq 1$} & \multicolumn{2}{|c|}{$\cdot, Y r V$} \\
\hline \multicolumn{4}{|c|}{ درحة الكفاعة للدويل } & & & \multicolumn{2}{|c|}{ qV,rq } & \multicolumn{2}{|c|}{$9 \cdot, 9 \leq$} & \multicolumn{2}{|c|}{$\wedge q, \varepsilon r$} \\
\hline
\end{tabular}

الإثارة (*) تشير إلى أن قيمة المعيار الأكبر أو الأصغر هي الأعظم أهمية للإطراف المستفيدة 


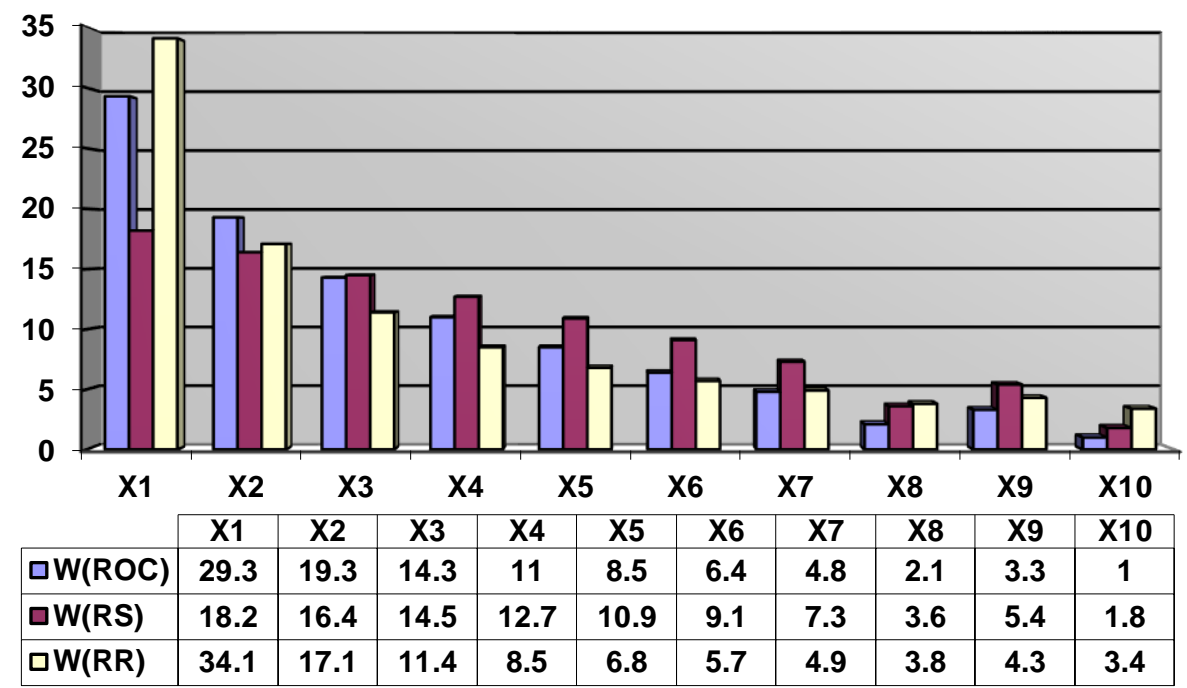

شكل رقم (1): مقارنة الاهمية النسبية لمعايير الاداء الانشائي.

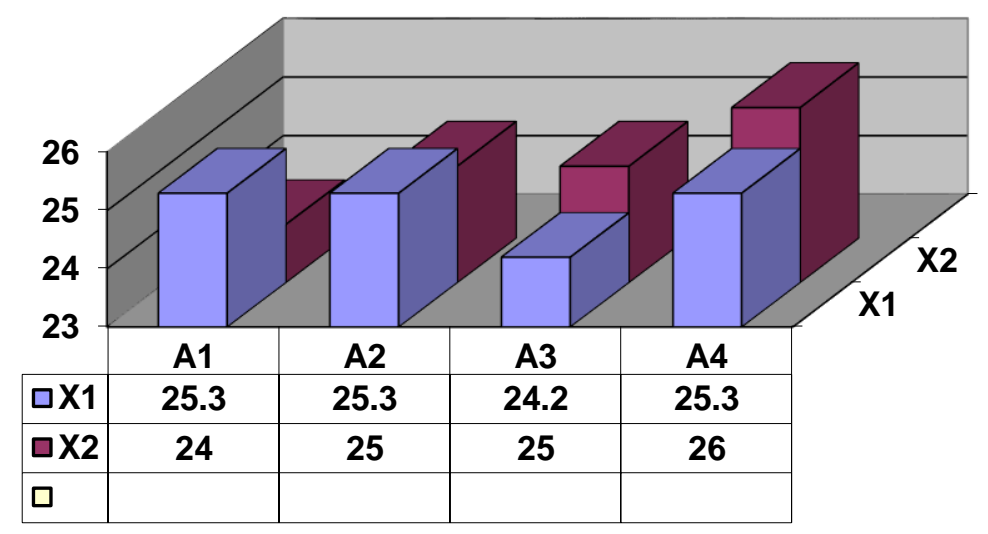

شكل رقم (Y): مقارنة الاهمية النسبية للمشاريع ضمن المعايير (X),XY) 


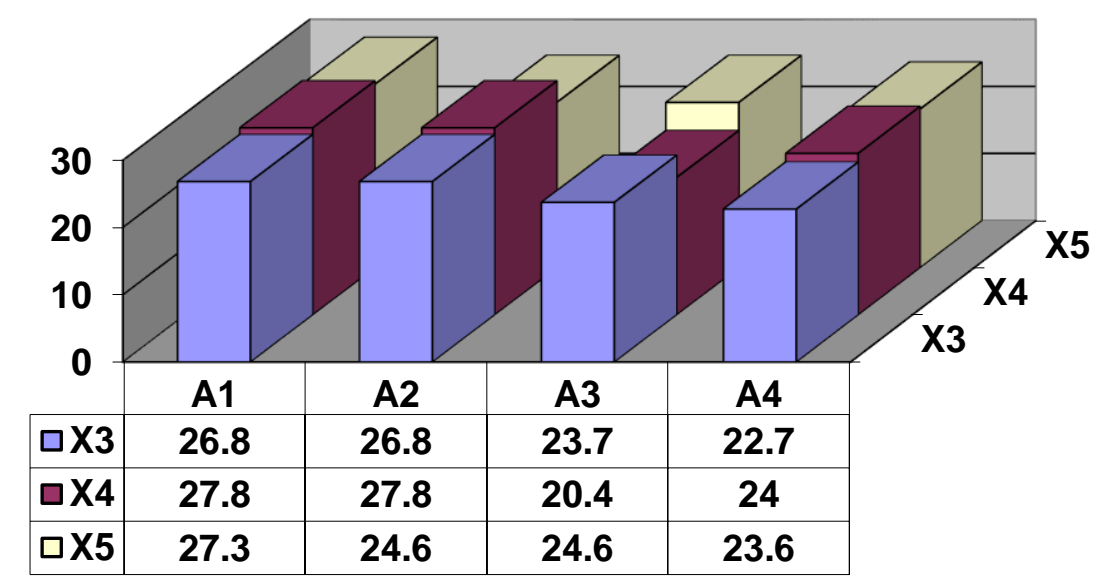

شكل رقم (r): مقارنة الاهمية النسبية للمشاريع ضمن المعايير (Xr,X气,X0)

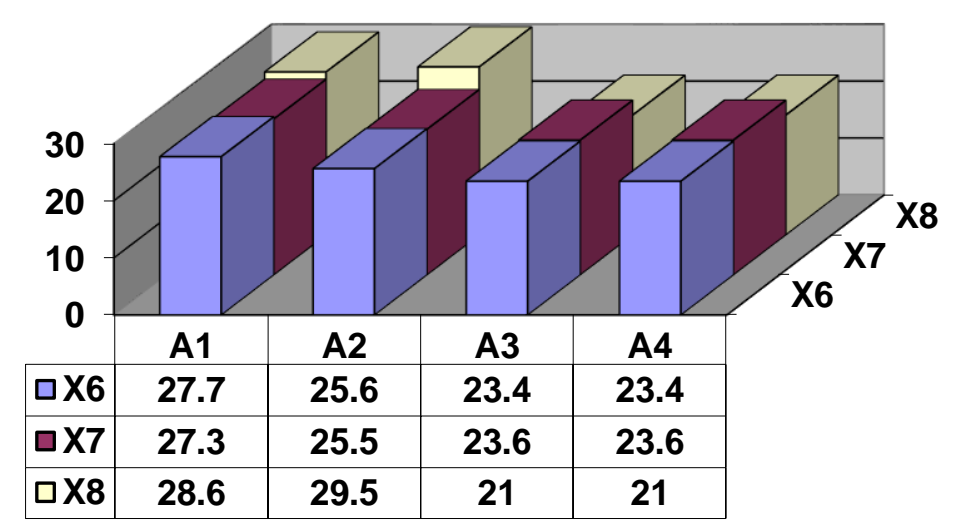

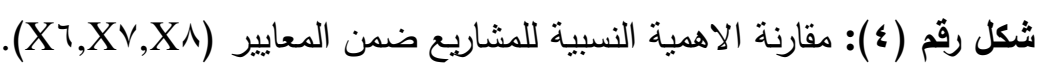

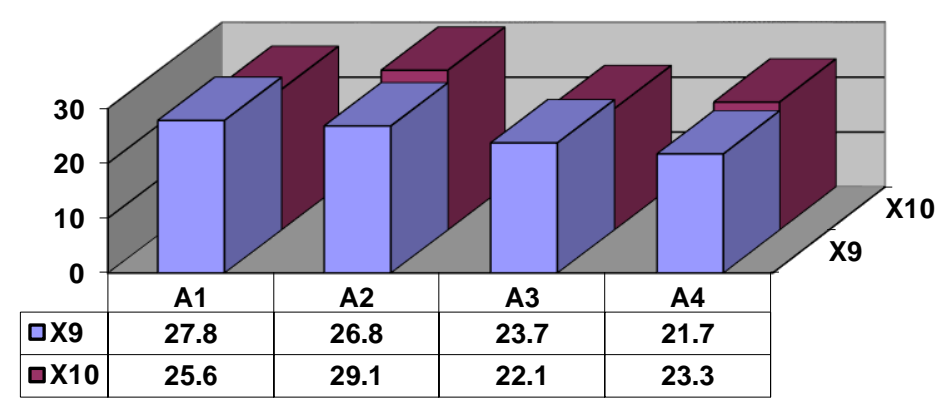

شكل رقم (•): مقارنة الاهمية النسبية للمشاريع ضمن المعايير (· • (X,X). 


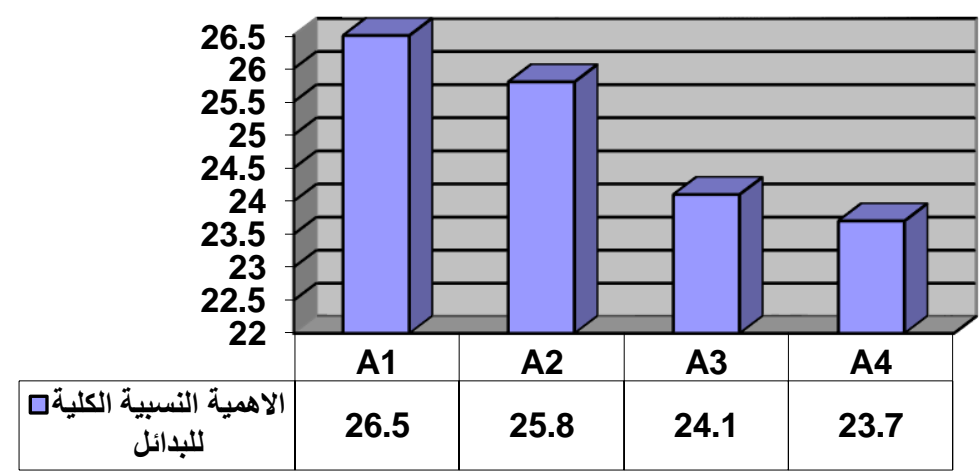

شكل رقم (†): مقارنة الاهمية النسبية الكلية للمشاريع لجميع المعايير.

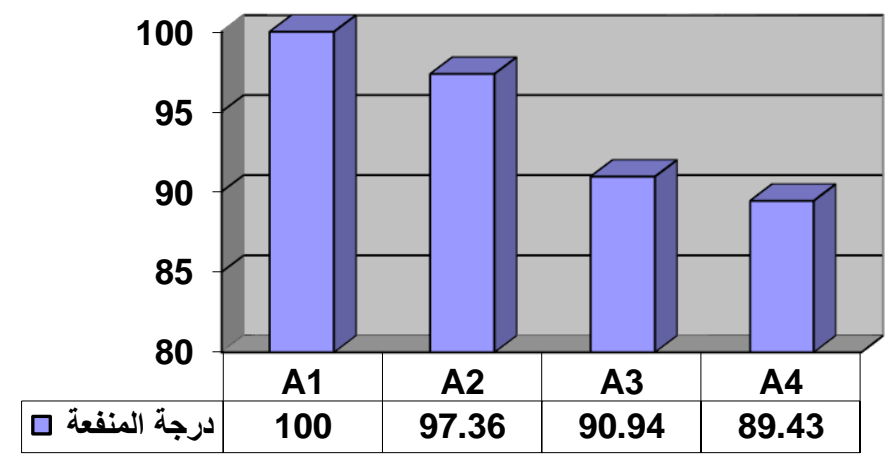

شكل رقم (V): مقارنة درجة المنفعة للمشاريع لجميع المعايير. 


\section{ملحق (1)}

الاستبيان الميداني

أولا :- يرجى بييان القيمة النوعية الدنيا (L) والعليا (U) لكل بديل من بدائل المشاريع مقابل كل معيار من معايير الأداء الإنشائي، وذللك بوضع الرمز المناسب في أسفل كل معيار •

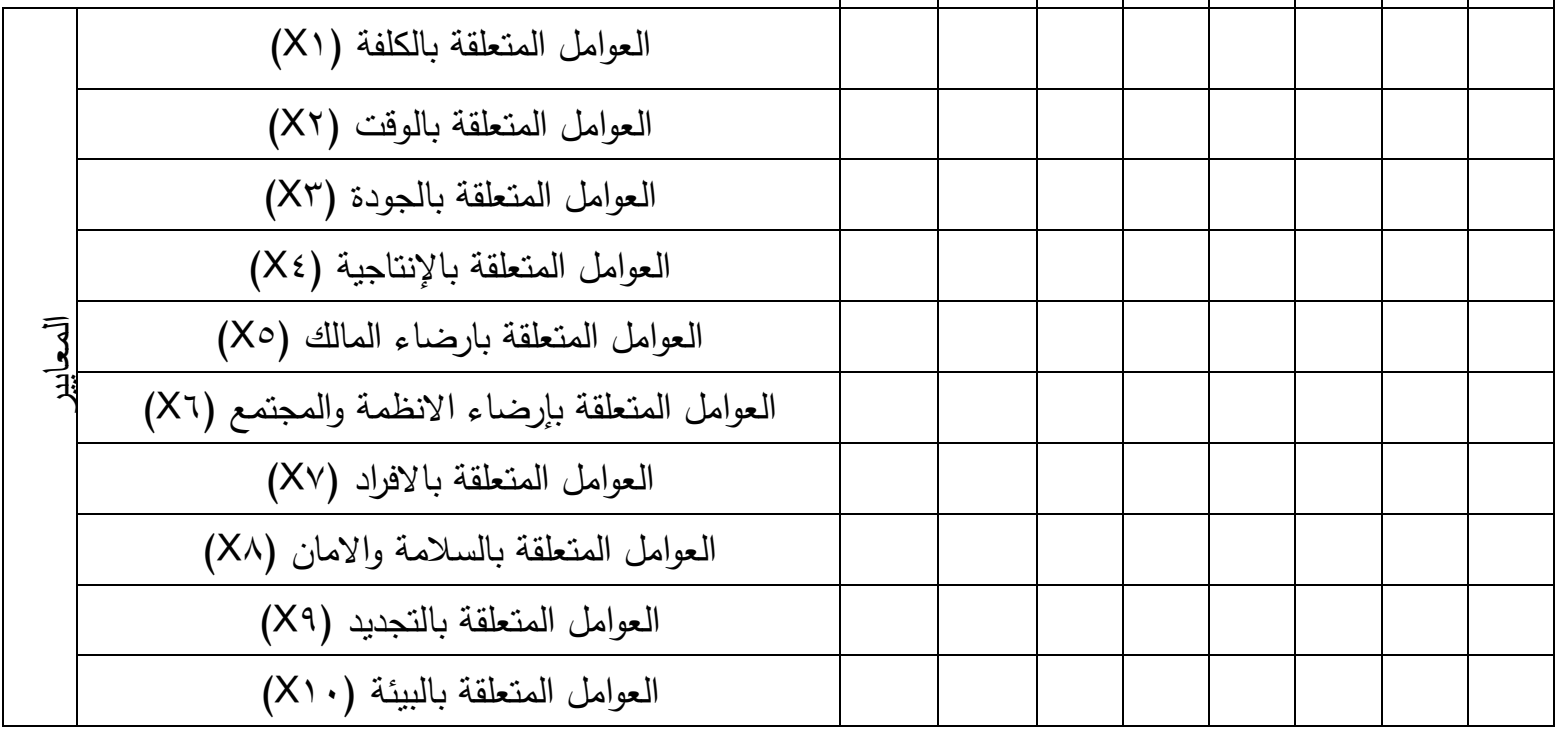

مصفوفة القرار لمعايير التقييم للاداء الانثائي للمشاريع بعليع

\begin{tabular}{|c|c|c|c|c|c|c|c|c|c|}
\hline AAA & AA & A & BBB & BB & B & CCC & CC & C & D \\
\hline $1 .$. & $\uparrow \cdot$ & $\Lambda \cdot$ & $V \cdot$ & $\ddots \cdot$ & 0. & $\varepsilon \cdot$ & $r \cdot$ & $r \cdot$ & 1. \\
\hline
\end{tabular}

قيم معايير التقييم

حيث يمثل الرمز AAA التقييم النوعي امنياز والذي تساوي قيمته . . . وهكذا بالنسبة لبقية الرموز ويوضح المثال التالي كيفية ملا الجدول في الاستمارة. .ضع القيمة النوعية لمعيار الكلفة مقابل البديل مشروعا ؟ ؟ فاذا V. وضعت الرمز BBB

\begin{tabular}{|c|c|c|c|c|}
\hline & \multicolumn{4}{|c|}{ المشاريع } \\
\hline & مشروعا & مشروعr & مشروعَ & مشروعء \\
\hline الكلفة & BBB & & & \\
\hline
\end{tabular}

ثانيا :-يرجى بيان ترتيب المعايير وفقا لأهمية وتأثير كل معيار من المعايير على الاداء الانشائي للمشروع وذلك بوضع التزتيب المناسب في اسفل كل معيار

\begin{tabular}{|l|l|l|l|l|l|l|l|l|l|}
\hline$(X 1 \cdot)$ & $(X 9)$ & $(X \wedge)$ & $(X \vee)$ & $(X \uparrow)$ & $(X 0)$ & $(X \varepsilon)$ & $(X Y)$ & $(X Y)$ & $(X)$ \\
\hline & & & & & & & & & \\
\hline
\end{tabular}


تقييم الأداء الإنشائي لمشاريع محافظة ديالى باستخدام تقتية تقييم البدائل المتعددة المعايير النسبية المعقدة بعلاقات رمادية (COPRAS-G)

\title{
STRUCTURAL PERFORMANCE EVALUATION OF THE DIYALA CITY PROJECTS USING THE METHOD OF MULTIPLE CRITERIA COMPLEX PROPORTIONAL ASSESSMENT OF ALTERNATIVES WITH GREY RELATIONS (COPRAS-G)
}

\author{
Hafeth I. Naji \\ Lecturer \\ College of Engineering \\ Diyala University/ Iraq
}

\begin{abstract}
The construction sector the main driver of the national economy on the other hand is facing this sector stumbled in his performance and the advantage of the merger of several problems at the same time due to the complex nature and uncertain environment of the project and the multiplicity of factors that lead to the occurrence of a time delay in implementation and high cost of the project, and to address this challenges requires the project management methods in this area and the potential of a smart and distinctive in making the right decisions. The method of multiple criteria complex proportional assessment of alternatives with Grey relations (COPRAS-G) is one of those methods. This research focuses on providing (COPRAS-G) technique helps to make the best decisions when setting up the project, which encountered problems are complex and multi-criteria (MCDM). And apply this technique on a variety of areas as it allows the comprehensive study and nonbiased criteria and alternatives. This research aims to highlight the importance of structural performance, and how it can be employed technical grounds (COPRAS-G) in assessing the structural performance of the Diyala City projects, through the identification of the criteria used to select the best project in terms of structural performance, and also to identify a list of projects the main province in order to choose the project optimization of this area. For the purpose of achieving the objective of research, has been collecting its own data from the literature that dealt with a multiple criteria complex proportional assessment of alternatives with Grey relations and, structural performance, and finally personal interviews of qualified designers and implementers of these projects. The results of data analysis for the sample showed that standards of cost, time, and quality is the most important criteria for bilateral comparisons between projects, and that the standard cost is more important than the rest of the criteria in the projects. Finally, and by calculating the relative importance of the projects and priorities of the alternatives and the utility degree have, we find that plant project streams
\end{abstract}


Baquba has received the largest share of the benefit and importance compared with other projects.

In the end, was a set of conclusions and recommendations of the various aspects of the topic from, accelerate the application of techniques of decision-making multi-criteria in the evaluation of projects in addition to expediting the application of the proposed systems for Structural Performance by researchers to help corporate managers that the decision-making efficient, which will lead to a process control and more effective control on the construction project.

Key words: - Structural performance, COPRAS-G 